\title{
EXISTENCE OF ENDO-RIGID BOOLEAN ALGEBRAS
}

In [Sh 2] we, answering a question of Monk, have explicated the notion of "a Boolean algebra with no endomorphisms except the ones induced by ultrafilters on it" (see $\S 2$ here) and proved the existence of one with character density $\aleph_{0}$, assuming first $\diamond_{\aleph_{1}}$ and then only $C H$. The idea was that if $h$ is an endomorphism of $B$,not among the "trivial" ones, then there are pairwise disjoint $D_{n} \in B$ with $h\left(d_{n}\right) \not \subset d_{n}$. Then we can, for some $S \subset \omega$, add an element $x$ such that $d_{n} \in B$ with $h\left(d_{n}\right) \not \subset d_{n} \dot{i}$ Then we can, for some $S \subset \omega$, add an element $x$ such that $d \leq x$ for $n \in S, x \cap d_{n}=0$ for $n \notin S$ while forbidding a solution for $\left\{y \cap h\left(d_{n}\right): n \in S\right\} \cup\left\{y \cap h\left(d_{n}\right)=\right.$ $0: n \notin S\}$. Further analysis showed that the point is that we are omitting positive quantifier free types. Continuing this Monk succeeded to prove in $Z F C$, the existence of such Boolean algebras of cardinality $2^{\aleph_{0}}$. In his proof he

(a) replaced some uses of the countable density character by the $\aleph_{1}$ chain condition

(b) generally it is hard to omit $<2^{\aleph_{0}}$ many types but because of the special character of the types and models involve, using $2^{\aleph_{0}}$ almost disjoint subsets of $\omega$, he succeeded in doing this

(c) for another step in the proof (ensuring indecomposability - see Definition 2.1) he ( and independently by Nikos) found it is in fact easier to do this when for every countable $I \subset B$ there is $x \in B$ free over it.

The question of the existence of such Boolean algebras in other cardinalities open (See [DMR] and a preliminary list of problems for the handbook of Boolean Algebras by Monk).

We shall prove (in $Z F C$ ) the existence of such $B$ of density character $\lambda$ and cardinality $\lambda^{\aleph_{0}}$ whenever $\lambda>\aleph_{0}$. We can conclude answers to some other questions from Monk's list, (combine 3.1 with 2.5). We use a combinatorial method from [Sh 3], [Sh 4], that is represented in section 1.

In [Sh 1], [Sh 6] (and [Sh 7]) the author offers the opinion that the combinatorial proofs of [Sh 1], Ch VIII (applied thee for general first order theories) should be useful for proving the existence of many non-isomorphic, ad/or pairwise non-embeddable structures which have few (or no) automorphisms or endomorphism of direct decompositions etc. As an illumination, in [Sh 6] a rigid Boolean algebra in every $\lambda^{\aleph_{0}}$ omitting countable types along the way, the method is proved in $Z F C$, nevertheless it has features of the diamond. It has been used (so in Gobel and Corner [CG] and Gobel and Shelah [GS1],[GS2]. See more on the method and on refinements of it in [Sh 4] and [Sh 3] and mainly [Sh 5]. 
Content Let $\lambda>k$ be fixed infinite cardinal

We shall deal with the case $c f \lambda \aleph_{0}, \lambda^{\aleph_{0}}=\lambda^{k}$, and usually $k=\aleph_{0}$. Let $\mathcal{M}$ be a function symbols, each with $\leq k$ places, of power $\leq \lambda$. Let $\zeta(\lambda, k)$ be the vocabulary with function symbols $\{F i, j: i<\lambda j<k\}$ where $F_{i}, j$ is a $j$-place function symbol. Let $\mathcal{M}=\mathcal{M}_{\lambda, k}(T)$ the free $\zeta(\lambda, k)$-algebra generated by $T \stackrel{\text { def }}{=} k>\lambda(=\{\eta: \eta$ a sequence $<k$ of ordinals $<\lambda)$ (We could have as well considered $T$ as a set of urelements, and let $\mathcal{M}$ be the family $H_{<k^{\prime}}(T)$ of sets hereditarily of cardinality $<k$ build from urelements].

1.2 Explanation. We shall let $B_{0}$ be the Boolean Algebra freely generated by $\{\eta: \eta \in T\}, B_{0}^{C}$ its completion and we can interprete $B_{0}^{c}$ as a subset og $\mathcal{M}$ (each $a \in B_{0}^{c}$ has the form $\cup_{\eta<\omega} \tau_{\eta}$ where $\tau_{\eta}$ is a Boolean combination of members of $T$, so as we have in $L \aleph_{0}$-place function symbols there is no problem). As the $\eta \in T$ may be over-used we replaced them for this purpose by ?? (e.g. let $F \in L$ be a monadic function symbol, $x_{\eta}=F(\eta)$ ).

Our desired Boolean Algebra $B$ will be a subalgebra of $B_{0}^{c}$ containing $B_{0}$.

\subsection{Definition.}

(1) Let $L_{n}$, for $n<w$, be fixed vocabularies (= signatures), $\left|L_{n}\right| \leq$ $L_{n} k, L_{n+1} \subset L_{n+1}$, (with each predicate function symbol finitary for simplicity) let $P_{n} \in L_{n+1}-L_{n}$ be monadic predicates.

(2) Let $\mathcal{F}_{n}$ be the family of sets (or sequences) of the form $\left\{\left(f_{\ell}, N_{\ell}\right)\right.$ : $\ell \leq\}$ satisfying

a) $f_{\ell}:{ }^{\ell \geq k} \rightarrow T$ is a tree emdedding $\mathrm{i}$.

(i) $f_{\ell}$ is length preserving i.e. $\eta, f_{e} l l(\eta)$ have the same length.

(ii) $f_{\ell}$ is order preserving i.e. for $\eta, \nu \in^{\ell \leq k}, \eta<\nu$ iff $f_{\ell}(\eta)<f_{\ell}(\nu)$

b) $f_{e+1}$ extend $f_{\ell}($ when $\ell+1 \leq n)$

c) $N_{\ell}$ is an $L_{\ell^{\prime}}^{\prime}$ model of power $\leq k,\left|N_{\ell}\right| \subset|X X|$, where $L_{\ell}^{\prime} \subset L_{\ell}$.

d) $L^{\prime} \ell+1 \cap L_{\ell}=L_{\ell}^{\prime}$ and $N_{\ell+1} \uparrow L^{\prime} \ell$ extends $N_{\ell}$

e) if $P_{m} \in L_{m+1}^{\prime}$, then $P_{m}^{N_{\ell}}=\left|N_{m}\right|$ when $m<\ell \leq n$ and

f) $\operatorname{Rang}\left(f_{\ell}\right)-\cup_{m<\ell} \operatorname{Rang}\left(f_{m}\right)$ is included in $N_{\ell}\left|-\cup_{m<\ell}\right| N_{m} \mid$.

(3) Let $\mathcal{F}_{w}$ be the family of pairs $(f, N)$ such that for some $\left(f_{\ell}, N_{\ell}\right)(\ell<$ $\omega)$ the following holds:

(i) $\left\{\left(f_{\ell}, N_{\ell}\right): \ell \leq n\right\}$ belongs to $\mathcal{F}_{n}$ for $n<w$.

(ii) $f=\cup_{\ell<\omega} f_{\ell}, N=\cup_{n<w} N_{n}$, (i.e. $|N|=\cup_{n<\omega}\left|N_{n}\right|, L(N)=$ $\cup_{n} L\left(N_{n}\right)$, and $N \uparrow L\left(N_{n}\right)=\cup_{n<m<\omega} N_{m} \uparrow L\left(N_{m}\right)$

(4) For any $(f, N) \in \mathcal{F}_{\omega}$ let $\left(f_{n}, N_{n}\right)$ be as above (it is easy to show that $\left(f_{n}, N_{n}\right)$ is uniquely determined - notice d),e) in (2), ) so for $\left(f^{\alpha}, N^{\alpha}\right)$ we get $\left(f_{n}^{\alpha}, N_{n}^{\alpha}\right)$

(5) A branch of Rang $(f)$ or of $f$ (for $f$ as in (3)) is just $\eta \in{ }^{\omega} \lambda$ such that for every $n<\omega, \eta\lceil n \in \operatorname{Rang}(f)$. 
We will be given $W=\left\{\left(f^{\alpha}, N^{\alpha}\right): \alpha<\alpha\left(^{*}\right)\right\}$, so that every branch $\eta$ of $f^{\alpha}$ converges to some $\zeta(\alpha), \zeta(\alpha)$ non decreasing (in $\alpha$ ). We have a free object generated by $T\left(B_{0}\right.$ in our case $)$ and by induction on $\alpha$ we define $B_{\alpha}$ and $a_{\alpha}, B_{\alpha}$ increasing continuos, such that $B_{\alpha+1}$ is an extension of $B_{\alpha}, a_{\alpha} \in B_{\alpha+1}-B_{\alpha}$ usually $B_{\alpha+1}$ is generated by $B_{\alpha}$ and $a_{\alpha}$ is in the completion of $\left.B_{0}\right)$. Every element will depend on few $(\leq k)$ members of $T$, and $a_{\alpha}$ "depends" in a peculiar way: the set $Y_{\alpha} \subseteq T$ on which it "depends" is $Y_{\alpha}^{0} \cup Y_{\alpha}^{1}$ where $Y_{\alpha}^{0}$ is bounded below $\zeta(\alpha)$ (i.e. $Y_{\alpha}^{0} \subseteq{ }^{\omega>} \zeta$ for some $\zeta<\zeta(\alpha))$ and $Y_{\alpha}^{1}$ is a branch of $f^{\alpha}$ or something similar. See more in 1.8.

1.5 Definition of the Game. We define for $W \subseteq \mathcal{F}_{\omega}$ a game $G m(W)$, which asts $\omega$-moves.

In the $n$-th move:

Player II: Choose $f_{n}$, a tree-embedding of $n \geq \lambda$, extending $\cup_{\ell<n} f_{\ell}$, such that $\operatorname{Rang}\left(f_{n}\right)-\cup_{\ell<n} \operatorname{Rang}\left(f_{\ell}\right)$ is disjoint to $\cup_{\ell<n}\left|N_{\ell}\right|$; then

player $I$ chooses $N_{n}$ such that $\left\{\left(f_{\ell}, N_{\ell}\right): \ell \leq n\right\} \in \mathcal{F}_{n}$.

In the end player II wins if $\left(\cup_{n<\omega} f_{n}, \cup_{n<\omega} N_{n}\right) \in W$.

1.6 Remark. We shall be interested in $W$ such that player II wins (or at least does not lose) the game, but $W$ is "thin". Sometimes we need a strengthening of the first player in two respects: he can force (in the $n$-th move) $\operatorname{Rang}\left(f_{n+1}\right)-\operatorname{Rang}\left(f_{n}\right)$ to be outside a "small" set, and in the zero move he can determine an arbitrary initial segment of the play.

1.7 Definition. We define, for $W \subseteq \mathfrak{F}_{\omega}$, a game $G M^{\prime}(W)$ which lasts $\omega$-moves.

In the zero move

player II choose $f_{0}$, a tree embedding of $0 \geq$ to ${ }^{0 \geq \lambda}$ (but there is only one choice).

player $I$ chooses $k<\omega$ and $\left\{\left(f_{\ell}, N_{\ell}\right): \ell \leq\right\} \in \mathfrak{f}_{k}$, and $X_{0} \subset T,\left|X_{0}\right|<\lambda$. In the $n$-th move, $n<0$ :

player $I I$ chooses $f_{k+n}$ a tree embedding of $(k+n) \geq k$ into $(k+n) \geq \lambda$, with Range $f_{k+n}-\cup_{\ell<k+n} R$ ang $f_{\ell}$ disjoint to $\cup_{\ell<k+n} N_{\ell} \cup \cup_{\ell<n} X_{\ell}$

player $I$ choose $N_{k+n}$ such that $\left\{\left(f_{\ell}, N_{\ell}\right): \ell \leq k+n\right\} \in \mathfrak{F}_{k+n}$ and $X_{n} \subseteq T,\left|X_{n}\right|<\lambda$

1.8 Remark. What do we want from $W$ ?: First that by adding an element (to $\left.B_{0}\right)$ for each $(f, N)$ we can "kill" every undesirable endomorphism, for this it has to encounter every possible endomorphism, and this will be served by "W a barrier". For this $W=\mathcal{F}_{\omega}$ is O.K. but we also want $W$ to be thin enough so that various demands will have small interaction, for this disjointness and more are demanded.

\subsection{Definition.}

(1) We call $W \subseteq \mathcal{F}_{\omega}$ a strong barrier if player II wins in $g m(W)$ and even $G m^{\prime}(W)$ (which just means he has a winning strategy.) 
(2) We call $W$ a barrier if player I does not win in $G m(W)$ and even does not win in $G m^{\prime}(W)$.

(3) We call $W$ disjoint if for any distinct $\left(f^{\ell}, N^{\ell}\right) \in W(\ell=1,2), f^{1}$ and $f^{2}$ have no common branch.

\subsection{The Existence Theorem.}

(1) if $\lambda^{\aleph_{0}}=\lambda^{k}, c f \lambda>\aleph_{0}$ then there is a strong disjoint barrier.

(2) Suppose $\lambda^{\aleph_{0}}=\lambda^{k}$, cd $\lambda>\aleph_{0}$. Then there is $W=\left\{\left(f^{\alpha}, N^{\alpha}\right): \alpha<\right.$ $\left.\alpha^{*}\right\} \subseteq \mathcal{F}_{w}$ and a function $\zeta: \alpha^{*} \rightarrow \lambda$ such that:

(a) $W$ is a strong disjoint barrier, moreover for every stationary $S \subset\left\{\delta<\lambda:\right.$ cf $\left.\delta=\aleph_{0}\right\}\left\{\left(f^{\alpha}, N^{\alpha}\right): \alpha<\alpha^{*}, \zeta(\alpha) \in S\right\}$ is a disjoint barrier. $\quad$ (b) $\operatorname{cf}(\zeta(\alpha))=\aleph_{0}$ for $\alpha<\alpha^{*}$. $\quad$ (c) Every branch of $f^{\alpha}$ is an increasing sequence converging to $\zeta(\alpha)$. (d) If $\bar{\eta}$ is a sequence from $T$ (of any length $\left.\gamma<k^{*}\right), \tau(\bar{x})$ a term of Land every $X_{i}$ really appears, $\lg (\bar{x})=\gamma$ and $\tau(\bar{\eta}) \in N^{\alpha}$ then $\bar{\eta} \subseteq N^{\alpha} \cap T$. (e) If $\zeta(\beta)=\zeta(\alpha), \beta+k^{\aleph_{0}} \leq \alpha<\alpha^{*}$ and $\eta$ is a branch of $f^{\alpha}$ then $\eta \uparrow k \notin N^{\beta}$ or some $k<w$. (f) If $\lambda=\lambda^{k}$ we can demand: if $\eta$ is a branch of $f^{\alpha}$ and $\eta \uparrow k \in N^{\beta}$ for all $k<\omega$ (where $\alpha, \beta<\alpha^{*}$ ) then $N^{\alpha} \subseteq N^{\beta}$ (and even??? $N_{n}^{\alpha} \in N^{\beta}$ if $\mathcal{M}=H_{<k^{+}}(T)$ ).

\section{REFERENCE}

\section{Preliminaries on Boolean Algebras}

We review here some easy material from [Sh 2].

\subsection{Definition.}

(1) For any endomorphism $h$ of a Boolean Algebra $B$. let $E X \operatorname{Ker}(h)=$ $\left\{x_{1} \cup x_{2}: h\left(x_{1}\right)=0\right.$, and $h(y)=y$ for every $\left.y \leq x_{2}\right\}$.

$\operatorname{Ex} \operatorname{Ker}^{*}(h)=\left\{x \in B:\right.$ in $B / E x K \operatorname{Ker}(h)$, below $x / \operatorname{Ex} \operatorname{Ker}^{*}(h)$, there are only finitely many elements $\}$.

(2) A Boolean algebra is endo-rigid if for every endomorphism $h$ of $B, B / \operatorname{Ex} \operatorname{Ker}(h)$ is finite (equivalently: $1_{B} \in \operatorname{Ex} \operatorname{Ker}(h)$ ).

(3) A Boolean algebra is indecompensable if there are no two disjoint ideal $?_{0}, I_{1}$ of $B$, each with no maximal member which generate a maximal ideal $\left\{a_{0} \cup a_{1}: a_{0} \in I_{0}, a_{1} \in I_{1}\right\} 0$.

(4) A Boolean algebra $B$ is $\aleph_{1}$-compact if for pairwise disjoint $?_{n} \in$ $B(n<\omega)$ for some $x \in B, x \cap d_{2 n+1}=0, x \cap d_{2 n}=d_{2 n}$.

\subsection{Lemma.}

(1) A Boolean algebra $B$ is endo-rigid iff for every endomorphism of $B$ is the endomorphism of some scheme (see Definition 2.3 below).

(2) A Boolean algebra $B$ is endo-rigid and indecomposable iff every endomorphism of $B$ is the endomorphism of some simple-scheme (see Def 2.3 below). 
EXISTENCE OF ENDO-RIGID BOOLEAN ALGEBRAS

\subsection{Definition.}

(1) A scheme of an endomorphism of $B$ consists of a partition $a_{0}, a_{1}, b_{0}, \ldots, b_{n-1}$, $c_{0}, \ldots, c_{m-1}$ of $B$ of maximal nonprincipal ideal $I_{\ell}$ below $b_{\ell}$ for $\ell<n$, nonprincipal disjoint ideals $I_{\ell}^{0}, I_{\ell}^{1}$ below $c_{\ell}$ for $\ell<m$, a number $k<n$, and a partition $b_{0}^{*}, \ldots, b_{n-1}^{*}, c_{0}^{*}, \ldots, c_{m-1}^{*}$ of $a_{0} \cup$ $b_{0} \cup, \cdots \cup b_{k-1}$. We assume also that $\left[k+m>0 \Rightarrow a_{0}=0\right],[(n-$ $k)+m>0 \Rightarrow a_{1}=0$ ] and except in those cases there are no zero elements in the partition.

(2) The scheme is simple if $m-0$.

(3) The endomorphism of the scheme is the unique endomorphism $T$ : $B \rightarrow$ such that: $\quad$ (i) $T z=0$ when $x<a_{0}$ or $x \in I_{\ell}, \ell<k$, or $x \in I_{\ell}^{0}, \ell<m$.

(ii) $T z=x$ when $x \leq a_{1}$ or $x \in I_{\ell}, k \leq \ell<n$ or $x \in I_{\ell}^{1}, \ell<m$.

(iii) $T\left(b_{\ell}\right)=b_{\ell}^{*}$ when $\ell<k$.

(iv) $T\left(b_{\ell}\right)=b_{\ell} \cup b_{\ell}^{*}$ when $k \leq \ell<n$.

(v) $T\left(C_{\ell}\right)=c_{\ell} \cup c_{\ell}^{*}$ when $\ell<m$.

2.4 Claim. If $h$ is an endomorphism of a Boolean Algebra B, and B/Ex Ker $(h)$ is infinite then there are pairwise disjoint $d_{n} \in B(n>\omega)$ such that $h\left(d_{n}\right) \not \subset$ $d_{n}$. By easy manipulation we can assume that $h\left(d_{n}\right) \cap d_{n+1} \neq 0$, and if $B$ satisfies the c.c.c then $\left\{d_{n}: n<w\right\}$ is a maximal antichain. So, an endomorphism of a scheme is a "trivial" endomorphism generated by ideals.

\subsection{Lemma.}

(1) Every endo-rigid Boolean Algebra $B$ is a Hopfian and dual Hopfian. Even $B+B$ is Hopfian (and dual Hopfian) but not rigid.

Proof Easy to check using 2.2, 2.3.

\section{The Construction}

3.1 Main Theorem. Suppose $\lambda>\aleph_{0}$. Then there is a B.A. (Boolean Algebra) $B$ such that:

(1) B satisfies the c.c.c.

(2) $B$ has power $\lambda^{\aleph_{0}}$ and $T_{1}(B)=\lambda$ where $T_{1}$ is the density character.

(3) $B$ is endo-rigid and indecomposable.

Proof We concentrate on the case $c f(\lambda) \geq \aleph_{1}$ (on the case $c f \lambda=\aleph_{0}$ see $[$ Sh $5, \S 2, \S 3])$ we shall use Theorem 1.7, and let $W=\left\{\left(f^{\alpha}, N^{\alpha}\right): \alpha<\alpha^{*}\right\}$, the function $\zeta, \mathcal{M}$ and $T={ }^{\omega>} \lambda$ be as there.

We will think of the game as follows: player I tries to produce a non trivial endomorphisms $h$. Player II supplies (via range $\left(f_{1}\right)$ ) elements is 
$N_{i}$ in the language $L_{i}$ with a distinguished function symbol $h$ which is an endomorphism of Boolean Algebras. In the end, as $W$ is a strong barrier, we will get a model $N^{\alpha} \in W$, in the language $\cup_{i<\omega} L_{i}$ which includes a function $h$. We can think of $N^{\alpha}$ as a Boolean Algebra $\subseteq B_{0}^{c}$ with an endomorphism $h$.

Stage $\mathbf{A}$ Let $B_{0}$ be the B.A. freely generated by $\left\{x_{\eta}: \eta \in T\right\}$, let $x_{\eta}=a_{\eta}$ and $B_{0}^{c}$ be its completion. For $A \subseteq B_{0}^{c}$ let $\langle A\rangle_{B_{c}^{0}}$ can be represented as a countable union of members of $B_{0}$, so w.o.l.g. $B_{0}^{c} \subseteq \mathcal{M}$. We say $x \in B_{0}^{c}$ is based on $J \subseteq{ }^{>\lambda}$ if it is based on $\left\{x_{\nu}: \nu \in J\right\}$ [i.e. $x=\cup_{n} y_{n}$, each $y_{n}$ is in the subalgebra generated by $\left\{x_{\nu}: \nu \in J\right\}$ ] and let $\underline{d}(x)$ be the minimal such $J$. We shall now define by induction on $\alpha<\alpha^{*}$, the truth values of " $\alpha \in J$ ", $\eta_{\alpha}$, and members $\alpha_{\alpha}, b_{n}^{\alpha}, c_{m}^{\alpha}, d_{m}^{\alpha}, \tau_{m}^{\alpha}$ of $B_{0}^{c}$ such that, letting $B_{\alpha}=\left\langle B_{0}, \alpha_{i} i<\alpha, i \in J\right\rangle_{B_{0}^{c}}$ :

(1) $\eta_{\alpha}$ is a branch of $\operatorname{Rang}\left(f^{\alpha}\right), \eta_{\alpha} \neq \eta_{\beta}$ for $\beta<\alpha$

(2) if $\alpha \in J$, then for some $x i<\zeta(\alpha)$ :

$a_{\alpha}=\cup_{m}\left(\tau_{m}^{\alpha} \cap d_{m}^{\alpha}\right)$ where $\left\langle d_{m}^{\alpha}: m<\omega\right\rangle$ is a maximal antichain of non zero elements (of $\left.B_{0}^{c}\right) \cup_{m} \underline{d}\left(d_{m}^{\alpha}\right) \subseteq{ }^{\omega>} \xi, \tau_{m}^{\alpha} \in\left\langle x_{p}: \eta_{\alpha}\lceil m \leq\right.$ $p, p \in T\rangle_{B_{0}^{c}}$, and $\tau_{m}^{\alpha} \cap d_{m}^{\alpha}>0$.

(3) if $\alpha \in J$, then $b_{n}^{\alpha}, d_{n}^{\alpha} \in N_{0}^{\alpha}, c_{n}^{\alpha}, \tau_{m}^{\alpha} \in N^{\alpha}$ (hence each is based on $\left\{x_{\nu}: \nu \in{ }^{\omega>}, \nu \in N^{\alpha}\right\}$ ), and $b_{n}^{\alpha} \cap b_{m}^{\alpha}=0$ for $n \neq m$.

(4) for $\beta<\alpha, \beta \in J, B_{\alpha}$ omits $p_{\beta}=\left\{x \cap b_{n}^{\beta}=c_{n}^{\beta}: \eta<\omega\right\}$.

Remark. Many times we shall write $\beta<\alpha<\alpha^{*}$ or $w \subseteq \alpha<\alpha^{*}$ instead $\beta \in \alpha \cap J, w \subseteq \alpha \cap J$.

Before we carry the construction note:

3.2 Crucial Fact. : For any $x \in B_{\alpha}$ there are $k, \xi<\zeta$, and $\alpha_{0}<\cdots<\alpha_{k}$ such that $\zeta\left(\alpha_{0}\right)=\zeta\left(\alpha_{1}\right)=\zeta\left(\alpha_{2}\right)=\cdots=\zeta\left(\alpha_{k}\right)=\zeta, x$ is based on $\left\{x_{\nu}: \nu \in\right.$ $\omega>\xi$ or $\nu \in \underline{d}\left(\tau^{\alpha_{\ell m}}\right.$, for some $\left.\ell \geq k, m<\omega\right\}$.

Stage B Let us carry the construction. For $\xi<\lambda, w \subseteq \alpha^{*}$ let

$$
I_{\xi, w}=\left\{\nu: \nu \in{ }^{w>} \xi \text { or } \nu \in \cup_{m<w_{\gamma} \in w} \underline{d}\left(\tau^{\gamma_{m}}\right)\right\}
$$

We let $\alpha \in J$ iff $\left|N^{\alpha}\right| \subseteq B_{\alpha}, N^{\alpha}=\left(B_{0}^{c} \uparrow\left|N^{\alpha}\right|, h_{\alpha}\right)$ where $h_{\alpha}$ is an endomorphism of $B_{0}^{c} \uparrow\left|N^{\alpha}\right|$ hence maps $N_{n}^{\alpha}$ into $N_{n}^{\alpha}$ for $\left.n<\omega\right)$ and there are $d_{m}^{\alpha} \in N_{0}^{\alpha}$ for $m<\omega, d_{m}^{\alpha} \neq 0, d_{m}^{\alpha} \cap d_{\ell}^{\alpha}=0$ for $m \neq \ell$, such that for some $x i<\zeta(\alpha)$ each $d_{m}^{\alpha}$ is based on ${ }^{\omega>} \xi$, and there are a branch $\eta_{\alpha}$ of $\operatorname{Rang}\left(f^{\alpha}\right)$ and $\tau_{m}^{\alpha} \in N^{\alpha}(m<\omega)$ as in 1),2) above, such that if we add $\cup_{n<\omega}\left(\tau_{n}^{\alpha} \cap d_{\ell}^{\alpha}\right)$ to $B_{\alpha}$, each $p_{\beta}(\beta<\alpha$ is still omitted as well as $P_{\alpha}=\left\{x \cap h_{\alpha}\left(d_{m}^{\alpha}\right)=h_{\alpha}\left(d_{m}^{\alpha} \cap \tau_{m}^{\alpha}\right): m<\omega\right\}$ and $\left\langle d_{m}^{\alpha}: m<\omega\right\rangle$ is a maximal antichain.

If $\alpha \in J$ we choose $\eta^{\alpha}, d_{n}^{\alpha}, \tau_{m}^{\alpha}$, satisfying the above and let $b_{m}^{\alpha}=h_{\alpha}\left(d_{m}^{\alpha}\right), c_{m}^{\alpha}=$ $h_{\alpha}\left(d_{m}^{\alpha} \cap \tau_{m}^{\alpha}\right)$.

So " $\alpha \in J$ " means that I played Boolean Algebras and cadomorphisms as is the previous remark and we get in the Boolean Algebra with some 
The desired Boolean algebra $B$ is $B_{\alpha}^{*}$. We shall investigate it and eventually prove it is endo-rigid (in 3.11) and indecomposable (in 3.12) (3.1(1)), $3.1(2)$ are trivial).

Note also

\subsection{Fact.}

(1) For $\nu \in{ }^{\omega>} \lambda, x_{\nu}$ is free over $\left\{x_{n}: \eta \in^{\omega>} \lambda, \eta \neq \nu\right\}$ hence also over the subalgebra of $B_{0}^{c}$ of those elements based on $\left\{x_{\eta}: \eta \in{ }^{\omega>} \lambda, \eta \neq\right.$ $\nu\}$.

(2) For every branch $\eta$ of $F^{\alpha}$ such that $\eta \neq \eta_{\beta}$ for $\beta<\alpha, \xi(\alpha)$; and finite $\omega \subseteq \alpha$ there is $k$ such that $\{\rho: \eta \uparrow k \leq \rho \in T\}$ is disjoint to $W>\xi \cup \cup\left\{N^{\beta} \cap T: \beta \in w, \beta+2^{\aleph_{0}} \leq \alpha\right\} \cup \cup\left\{\bar{d}\left(\tau_{n}^{\beta}\right): n<\omega \in w\right\}$

From 3.2 we can conclude:

3.4 Fact. If $\xi<\zeta(\beta), \beta<\alpha, I \subseteq T$ finite then every element of $B_{\alpha}$, based on $I \cup^{\omega>} \xi$ is in $B_{\beta}$.

\subsection{Notation.}

(1) Let $B^{\xi}$ be the set of $a \in B_{0}^{c}$ supported by ${ }^{w>} \xi$

(2) For $x \in B_{0}^{c}, \xi<\lambda$ let $\operatorname{pr}_{\xi}(x)=\cap\left\{a \in B^{\xi}: x \leq a\right\}$.

(3) For $\xi<\lambda$ let $\epsilon(\xi)=\operatorname{Min}\{\gamma: \zeta(\gamma)>\xi\}$.

(4) For $\gamma, \alpha^{*}$ let $B_{<\gamma>}=\left\langle\left\{x_{\eta}: \eta \in{ }^{\omega>} \zeta(\gamma)\right\} \cup\left\{a_{\beta}: \beta<\gamma\right\}\right\rangle$.

(5) For $\xi<\lambda$ let $B_{[\xi]}=\left\langle\left\{x_{\eta}: \eta \in{ }^{W>} \xi\right\} \cup\left\{a_{\beta}: \zeta(\beta) \geq \xi\right\}\right\rangle_{B_{0}^{c}}$.

\subsection{Fact.}

(1) $B^{\xi}$ is a complete Boolean subalgebra of $B_{0}^{c}$.

(2) $\operatorname{pr}_{\xi}(x)$ si well defined for $x \in B_{0}^{c}$

(3) if $\xi_{0}<\xi_{1}<\lambda, x \in B_{0}^{c}$ then $\operatorname{pr}_{\xi_{0}}\left(\operatorname{pr}_{\xi_{1}}(x)=p r_{\xi_{0}}(x)\right.$.

(4) If $\xi<\lambda, w \subseteq T$ is finite then the function $\operatorname{pr}_{\xi, w}(x)=\cap\left\{y \in\left\langle B^{\xi} \cup\right.\right.$ $\left.\left.\left\{x_{\nu}: \nu \in w\right\}\right\rangle: x \geq y\right\}$ is well defined.

\subsection{Fact.}

(1) For $x \in B_{\alpha^{*}}, \xi<\lambda$, the element $\operatorname{pr}_{\xi}(x)$ belongs to $B_{[\xi]}$

(2) for $x \in B_{\alpha^{*}}, \xi, \lambda, w \subseteq \omega>(\xi+1)$, the element $\operatorname{pr}_{\xi w}(x)$ belongs to $B(\omega>\xi, w)$.

Proof We prove this for $x \in B_{\alpha}$, by induction on $\alpha$ (for all $\xi$ )

Note that $p r_{\xi}\left(\cup_{\ell<n} x_{\ell}\right)=\cup_{\ell<n} p r_{\xi}\left(x_{\ell}\right)$.

Case i: $\alpha=0$, or even $(\forall \beta, \alpha)[\zeta(\beta) \leq \xi]$

Easy; if $x=\tau\left(0, \ldots, a_{n-1}, x_{\nu_{0}}, \ldots, x_{n u_{m-1}}\right)$ where $\tau$ is a Boolean term, $a-\ell \in B_{[x i]}, \nu_{\ell} \in \omega>\xi$; by the remarks above w.l.o.g $x=\cap_{\ell<n+m} \tau_{\ell}, \tau_{\ell}\left\{a_{\ell}, 1-\right.$ $\left.a_{\ell}\right\}$ when $\ell<n, \tau_{\ell} \in\left\{x_{\nu_{\ell-n}}, 1-x_{\nu_{\ell-n}}\right\}$ when $n \leq n+m$, and the sequence $\left\langle x_{\nu_{0}}, \ldots, x_{\nu_{n-1}}\right\rangle$ is with no repetition, then clearly $p r_{\xi}(x)=\cap_{\ell<n} \tau_{\ell} \in B_{[\xi]}$ 
Case ii: $\alpha$ limit.

Trivial as $B_{\alpha}=\cup_{\beta<\alpha} B_{\beta}$.

Case iii: $\alpha=\beta+1$

By the induction hypothesis w.l.o.g. $x \notin B_{\beta}$. As $x \in B_{\alpha}$ there are disjoint $e_{0}, e_{1}, e_{2} \in B_{\beta}$ such that $x=e_{0} \cup\left(e_{1} \cap a_{\beta}\right) \cup\left(e_{2}-a_{\beta}\right)$. It suffices to prove that $\operatorname{pr}_{\xi}\left(e_{0}\right), p r_{\xi}\left(e_{1} \cap a_{\beta}\right), p r_{\xi}\left(e_{2}-a_{\beta}\right) \in B_{[\xi]}$, the first is trivial and w.l.o.g. we concentrate on the second. There are $\xi_{0}<\zeta(\beta)$ and $k<\omega$ such that $e_{1}$ is based on $J \stackrel{\text { def }}{=}^{\omega>} \lambda-\left\{\rho: \eta_{\beta} \uparrow k \leq \rho \in \omega>\lambda\right\}$ and each $d_{n}^{\beta}(n<\omega)$ is based on ${ }^{\omega>} \xi_{0}$. By case i, we can assume $\xi<\zeta(\beta)$ hence w.l.o.g. $\xi<\xi_{0}$, and by the induction hypothesis and 3.6(3) it suffices to prove $p r_{\xi_{0}}\left(e_{1} \cap a_{\beta}\right) \in B_{[\xi]}$. W.l.o.g. $e_{1} \cap d_{m}^{\alpha}=0$ for $m<k$ and now clearly $p r_{\xi_{0}}\left(e_{1} \cap a_{\beta}\right)=e_{1}$ as $p r_{\xi_{0}}\left(e_{1} \cap d_{m}^{\alpha} \cap \tau_{m}^{\alpha}\right)=e_{1} \cap d_{m}^{\alpha}$ for $m \geq k$, (because $d_{m}^{\alpha}, e_{1}$ are based on $J,{ }^{\omega>} \xi_{0} \subseteq J$ and $\tau_{m}^{\alpha}$ is based on ${ }^{\omega>} \lambda-J$ and is $>0$ )

2) Same proof.

3.8 Lemma. Suppose $I, w$ satisfy:

$(*)_{I, w} I \subseteq{ }^{\omega>} \lambda, w \subseteq a^{*}, I$ is closed under initial segments, and for every $\alpha<\alpha^{*}$ if $\wedge_{m<\omega}\left(\eta_{\alpha} \uparrow m \in I\right)$ then $\tau_{m}^{\alpha}, d_{m}^{\alpha}$ are based on $i$ and belong to $B(I<w)$.

Then for any countable $C \subseteq B_{\alpha}^{*}$ there is a projection from $\langle B(i, w), C\rangle_{B_{0}^{c}}$ onto $B(I, w)$.

Proof We can easily find $I(*), w(*)$ such that $C \subseteq W(*), w \subseteq w(*) \subseteq$ $\alpha^{*},|w(*)-w| \leq \aleph_{0}, I \subseteq I(*) \subseteq{ }^{\omega>} \lambda,|I(*)-I| \leq \aleph_{0}$ and if $\alpha \in w(*)-w$, then $\left.\tau_{m}^{\alpha}, d_{m}^{\alpha} \in B(I *), w(*)\right)$. Let $w(*)-w=\left\{\alpha_{\ell}: \ell<\omega\right\}$, and we define by induction on $\ell$ a natural number $k_{\ell}<\omega$, such that the sets $\left\{\nu \in{ }^{\omega>} \lambda: \nu\right.$ appears is $\tau_{m}^{\alpha_{\ell}}$ for some $\left.m>k_{\ell}\right\}$ are pairwise disjoint to $I$. Now we can extend the identity on $B(I, w)$ to a projection $h_{0}$ from $B(I(*), w)$ onto $B(I, w)$ such that of $\ell<\omega, m>k_{\ell}$, then $h_{0}\left(\tau_{m}^{\alpha_{\ell}} \cap d_{m}^{\alpha_{\ell}}\right)=0$. Now we can define by induction on $\alpha \in(w(*)-w) \cup\{0, \lambda\}$ a projection $h_{\alpha}$ from $B(I(*), w \cup(w(*) \cap \alpha))$ onto $B(I, w)$ extending $h_{\beta}$ for $\beta<\alpha$ and $\beta \in$ $(w(*)-w \cup\{0\})$. For $\alpha=0$ we have it defined, for $\alpha=\lambda$ we get the conclusion, and in limit stages take the union. In successive stages there is no problem by the choice of $h_{0}$, and the $k_{\ell}$ 's).

3.9 Claim. If $B^{\prime}$ is an uncountable subalgebra of $B_{\alpha^{\prime}}$ then there is an antichain $\left\{d_{n}: n<\omega\right\} \subseteq B^{\prime}$ and for no $x \in B, x \cap d_{2 n}=0, x \cap d_{2 n+1}=d_{n+1}$ for every $n$ provided that

$\left(^{*}\right)$ no one countable $I \subseteq{ }^{\omega>} \lambda$ is a support for every $a \in B^{\prime}$.

Proof We now define by induction on $\alpha<\omega_{1}, d_{\alpha}, I_{\alpha}$, such that:

(i) $I_{\alpha} \subseteq{ }^{\omega>} \lambda$ is countable.

(ii) $\cup_{\beta<\alpha} I_{\beta} \subseteq I_{\alpha}$ and for $\alpha$ limit, equality holds.

(iii) $d_{\alpha} \in B^{\prime}$ is supported by $I_{\alpha+1}$

There is no problem with this. 
By (iii) for each $\alpha$ there are $\tau_{\alpha}^{0} \in\left\langle a_{\eta}: \eta \in I_{\alpha}\right\rangle_{B_{0}^{c}}, \tau_{\alpha}^{1}, \tau_{\alpha}^{2} \in\left\langle a_{\eta}: \eta \in\right.$ $\left.I_{\alpha+1}-I_{\alpha}\right\rangle_{B_{0}^{c}}$ such that $\tau_{\alpha}^{1} \cap \tau_{\alpha}^{2}=0, \tau_{\alpha}^{0} \cap \tau_{\alpha}^{1} \leq d_{\alpha}, \tau_{\alpha}^{0} \cap \tau_{\alpha}^{2} \leq 1-d_{\alpha}$.

By Fodor's lemma w.o.l.g. $\tau_{\alpha}^{0}=\tau^{0}$ (i.e. does not depend on $\alpha$ ). For each $\alpha$ there is $n(\alpha)<\omega$ such that

$\tau_{\alpha}^{0} \in\left\langle a_{\eta}: \eta \in I_{\alpha} \cap^{n(\alpha) \geq \lambda}\right\rangle_{B_{0}^{c}}, \tau_{\alpha}^{1}, \tau_{\alpha}^{2} \in\left\langle a_{\eta}: \eta \in\left(I_{\alpha+1}-I_{\alpha}\right) \cap{ }^{n(\alpha)} \geq \lambda\right\rangle_{B_{0}^{c}}$

Again by renaming w.o.l.g $n(\alpha)=n(*)$ for every $\alpha$. Let for $n<\omega, d^{n}=$ $d_{n}-\cup_{\ell<n} d_{\ell}, \tau^{n}=\tau^{0} \cap \cap_{\ell<n} \tau_{\ell}^{2} \cap \tau_{n}^{1}$, so easily $D^{n} \in B^{\prime},\left\langle d^{n}: n<\omega\right\rangle$ is an

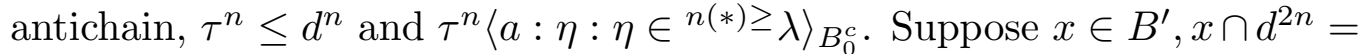
$0, x \cap d^{2 n+1}=d^{2 n+1}$. Hence for $n<\omega, x \cap \tau^{2 n}=0, x \cap \tau^{2 n+1}=\tau^{2 n+1}$. but by 3.8 (for $I={ }^{n(*)} \geq \lambda \omega=\phi$ and $c=\{x\}$ ), there is such $x$ in $\left\langle a_{\eta} ; \eta \in\right.$ $n(*) \geq \lambda\rangle_{B_{o}^{c}}$, an easy contradiction.

So we have proven that for every $\aleph_{1}$-compact $B^{\prime} \subseteq B_{\alpha^{*}}$, some countable $I \subseteq{ }^{\omega>} \lambda$ support every $x \in B^{\prime}$.

3.10 Claim. No infinite subalgebra $B^{\prime}$ of $B_{\alpha^{*}}$ is $\aleph_{1}$-compact.

Proof Suppose there is such $B^{\prime}$, and let $\xi$ be minimal such that there is such $B^{\prime} \subseteq B_{[\xi]}$

\section{Part I if $(*)$}

(a) $B^{\prime} \subseteq B_{\alpha^{*}}$ is $\aleph_{1}$-compact and infinite and

(b) $B^{\prime} \subseteq B_{[\xi]}$

then

(c) for every $\zeta<\xi$ and $x \in B^{\prime}-\left\{y:\left\{z \in B^{\prime}: z \leq y\right\}\right.$ is fintie $\}$, there is $x_{1} \in B^{\prime}, x-1 \leq x$ such that for no $y \in B_{[\zeta]}, y \cap x=x_{1}$.

So assume $B^{\prime}$, satisfies a) and b) but they fail c) for $\zeta<\xi$ and $x \in B^{\prime}$, where $\left\{y: y \leq x, y \in B^{\prime}\right\}$ is finite. So for every $z \in B^{\prime}$, there is $g(z) \in B_{[\zeta]}$ such that $g(z) \cap x=z \cap x$ (use $x_{1}=z \cap x$ ). Let $B^{a}$ be the subalgebra of $B_{[\zeta]}$ generated by $\left\{g(z): z \in B^{\prime}\right\}$. Clearly $\left\{y \in B^{\prime}: y \leq x\right\}=\left\{t \cap x: t \in B^{a}\right\}$. Let $x^{*}=p r_{\zeta}(x)$, (it is in $B_{[\zeta]}$ by 3.7(1)) and let $B^{b}=\left\{t \cap x^{*} ; t \in B^{a}\right\} \cup\left\{t \cup\left(1-x^{*}\right): t \in B^{a}\right\}$. Clearly $B^{b}$ is a subalgebra of $B_{[\zeta]}$, and $1-x^{*}$ is an atom of $B^{b} ; B^{b}$ is infinite as there are in $B^{\prime}$ distinct $x_{n} \leq x$, so $g\left(x_{n}\right) \in B^{a}$ hence $g\left(x_{n}\right) \cap x^{*} \in B^{b}$. As $x \leq x^{*}$ and $\left[n \neq \Rightarrow g\left(x_{n}\right) \cap x \neq g\left(x_{m}\right) \cap x\right]$ clearly $\left[n \neq m \Rightarrow g\left(x_{n}\right) \cap x^{*} /-g\left(x_{n}\right) \cap x^{*}\right]$. We shall prove that $B^{b}$ is $\aleph_{1^{-}}$ compact, thus contradicting the choice of $\zeta$. Let $d_{n} \in B^{b}$ be pairwise disjoint, and we want to find $T \in B^{b}, t \cap d_{2 n}=0, t \cap d_{2 n+1}=d_{2 n+1}$ (for $n<\omega$ ). Clearly w.o.l.g. $d_{n} \leq x^{*}$ (as $1-x^{*}$ is an atom of $B^{b}$ ). So $d_{n}=t_{n} \cap x^{*}$ for some $t_{n} \in B^{a}$, hence easily $t_{n} \cap x \in B^{\prime}$ so for some $x_{n} \in B^{\prime}, x_{n} \leq x$ and $t_{n} \cap x=x_{n} \cap x=x_{n}$. So $X-n=g\left(x_{n}\right) \cap x$.

For $n \neq m, x_{n} \cap x_{m}=\left(t_{n} \cap x\right) \cap\left(t_{m} \cap x\right) \leq\left(t_{n} \cap x^{*}\right) \cap\left(t_{m} \cap x^{*}\right)=$ d) $n \cap d_{m}=0$.

As $B^{\prime}$ is $\aleph_{1}$-compact there is $y \in B^{\prime}, y \cap x_{2 n}=0, y \cap x_{2 n+1}=$ $x_{2 n+1}$. Now $g(y), d_{n}, t_{N}$ belongs to $B_{[\xi]}$ and (as $x_{n} \leq x \leq x^{*}$ ):

(i) $g(y) \cap d_{2} n \cap x=g(y) \cap t_{2 n} \cap x=g(y) \cap x_{2 n} \cap x=y \cap x_{2 n} \cap x=0$.

(ii) $(y) \cap d_{2 n+1} \cap x=q(u) \cap t_{2 n+1} \cap x=q(u) \cap x_{2 n+1} \cap x=$ 
Now by the definition of $X^{*}=p r_{\zeta}(x),\left[\tau \in B_{[\zeta]} \wedge \tau \cap x=0 \Rightarrow \tau \cap\right.$ $\left.x^{*}=0\right]$. As $\left(1-\tau \in B_{[\zeta]}, x \leq 1-\tau\right)$ hence by (i) (for $\left.\tau=g(y) \cap d_{2 n}\right)$ : (iii) $g(y) \cap d_{2 n} \cap x^{*}=0$

Also by the definition of $x^{*}=p r_{\zeta}(x)$ :

$\tau_{1}, \tau_{2} \in B_{[\zeta]} \wedge \tau_{1} \cap x=\tau_{2} \cap x \Rightarrow x^{*}=\tau_{2} \cap x^{*}\left(\right.$ as $\tau_{1}-\tau_{2} \in B_{[\zeta]}, x \leq$ $\left.1-\left(\tau_{1}-\tau_{2}\right)\right)$ hence by (ii)

(iv) $g(y) \cap d_{2 n+1} \cap x^{*}=d_{2 n+1} \cap x^{*}$.

But $d_{n} \leq x *$, so from (iii) and (iv) $\left(g(y) \cap d_{2 n}=0,\left(g(y) \cap x^{*}\right) \cap\right.$ $d_{2 n+1}=d_{2 n+1}$, and $g(y) \in B^{a}$ hence $g(y) \cap x^{*} \in B^{b}$. So $B^{b}$ is $\aleph_{1}$-compact this contradicits the minimality of $\xi$, so we finish Part I.

Part II: If $B^{1}$ is $\aleph_{1}$-compact $B^{1} \subseteq B^{2}, B^{2}=\left\langle B^{1} \cup\{z\}\right\rangle$ then $B^{2}$ is $\aleph_{1}$-compact.

The proof is straightforward. [If $d_{n} \in B^{2}$ are pairwise disjoint, let $d_{n}=$ $\left(d_{n}^{1} \cap z\right) \cup\left(d_{n}^{2}-z\right)$ for some $d_{n}^{1}, d_{n}^{2} \in B^{1}$. Now w.l.o.g. $D_{n}^{1} \cap d_{m}^{1}=0$ for $n \neq m$ otherwise replace then by $d_{n}^{1}-\cup_{\ell<n} d_{\ell}^{1}$; similarly $d_{n}^{2} \cap d_{m}^{2}=0$, for $\neq m$. So there are $y^{\ell} \in B^{1}, y^{\ell} \cap d_{2 n+1}^{\ell}=d_{2 n+1}^{\ell}=d_{2 n+1}^{\ell}$, and $\left(y^{1} \cap z\right) \cup\left(y^{2}-z\right)$ is the solution.]

Part III: $\xi$ cannot be a successor ordinal.

Proof: Let $B^{\prime}$ satisfy $(*)$

Suppose $\xi=\zeta+1$, and by 3.9 there is a countable $I \subseteq \omega>\xi$ which support every $a \in B^{\prime}$. w.l.o.g. $I$ is closed under initial segments and $k=\mid I-\omega>\zeta$ is minimal. Now part I can be applied with $\left\langle B_{[\zeta]},\left\{a_{\eta}: \eta \in w\right\}\right\rangle_{B_{0}^{c}}$, for any finite $w \subseteq I$ of power $<k$ instead $B_{[\zeta]}$ (using 3.7(2) instead 3.7(1)). So by applying Part I (to $\left\langle B_{[\zeta]},\left\{a_{\eta}: \eta \in w\right\}\right\rangle_{B_{0}^{c}}$ ) we can add to its conclusion:

d) for every finite $w \subseteq I,|w|<\left|I-{ }^{\omega}>\zeta\right|$ and $x \in B^{\prime}$ and $x \in B^{\prime}$ for which $\left\{y \in B^{\prime}: y \leq x\right\}$ is infinite, there is $x_{1} \in B^{\prime}, x_{1} \leq x$ such that for no $y \in\left\langle B_{[\zeta]} \cup\left\{a_{\eta}: \eta \in w\right\}\right\rangle_{B_{0}^{c}}, y \cap x=x_{1}$.

Now $I-{ }^{\omega>} \zeta$ is infinite [otherwise let $\left.B^{\prime \prime}=\cup\left\{a_{\eta} ; \eta \in I-{ }^{\omega>} \zeta\right\}\right\rangle_{B_{o}^{c}}$, easily it is infinite and $\aleph_{1}$-compact by Part II and then we apply Part I: for

$I^{\omega>} \zeta=\left\{\eta_{0}, \ldots, \eta_{k-1}\right\}$ and for $u \subseteq\{0, \ldots, k-1\}$, let $x_{u} \stackrel{\text { def }}{=} \cap\left\{x_{\eta_{\ell}}: \ell \in\right.$ $u\} \cap\left\{1-x_{x \ell}: \ell<k, \ell \notin u\right\}$ so $x_{u} \in B ", 1=\cup\left\{x_{u}: u \subseteq\{0, \ldots, k-1\}\right\}$, hence for some $u,\left\{y \in B^{\prime}: y \leq x_{u}\right\}$ is infinite; $\zeta, x_{u}$ contradict the conclusion of Part I.

As $B^{\prime}$ is $\aleph_{1}$-compact, for any $x \in B^{\prime}$ such that $\{y \in \leq x\}$ is infinite, $x$ can be splitted in $B^{\prime}$ to two elements satisfying the same i.e. $x=x^{1} \cup$ $x^{2}, x^{1} \cap x^{2}=0,\left\{y \in B^{\prime}: y \leq x^{\ell}\right\}$ is infinite for the $\ell=1,2$. Let $I^{\omega>} \zeta=$ $\left\{\eta_{\ell}: \ell<\omega\right\}$, so we can find pairwise disjoint $e_{n} \in B^{\prime}$, such that $e_{n}=$ $d_{2 n} \cup d_{2 n+1}, d_{2 n} \cap d_{2 n+1}=0$ and that for no $y \in\left\langle B_{\left[B_{\zeta}\right]} \cup\left\{a_{n \ell}: \ell<n\right\}\right\rangle, y \cap$ $\left(d_{2 n} \cup d_{2 n+1}\right)=d_{2 n+1}$ for every $n$. So for no $n y \in\left\langle B[\zeta] \cup\left\{a_{n \ell}: \ell<n\right\}\right\rangle_{B_{0}^{c}}$.

As $y \in B^{\prime}$ clearly $y \in B_{[\zeta+1]}$, but $y$ is based on $\omega>\zeta \cup\left\{a_{\eta \ell}: \ell<\omega\right\}$ so 
Part IV: Let $B^{\prime}$, satisfy $\left(^{*}\right)$ of Part I. by 3.9 for some countable $I \subseteq \omega>\xi$, every $b \in B^{\prime}$ is based on $I$. By Part III $\xi$ is not a succesor ordinal, so necessarily $c f(\xi)=\aleph_{0}$, let $F i\left(B^{\prime}\right)=\left\{x \in B^{\prime}:\left\{y \in B^{\prime}: y \leq x\right\}\right.$ is finite $\}$. Next we shall show:

$\left.{ }^{* *}\right)$ for some finite $w \subseteq\{\gamma: \zeta(\gamma)=\xi\}$ and $x^{*} \in B^{\prime}-F i\left(B^{\prime}\right)$ for every $y<x^{*}$ from $B^{\prime}$, for some $Z \in\left\langle\cup_{\zeta<\xi} B[\zeta] \cup\left\{a_{\alpha}: \alpha \in w\right\}\right\rangle_{B_{0}^{c}}, z \cap x^{*}=y$.

Suppose $\left({ }^{* *}\right)$ fails and we define by induction on $n<\omega, x_{n}, y_{n}, w_{n}$, such that:

(i) $x_{n} \in B^{\prime}$,

(ii) $1-\cup_{i<n} x_{i} \notin F i\left(B^{\prime}\right)$

(iii) $w_{n} \subseteq\{\gamma: \zeta(\gamma)=\xi\}$ is finite

(iv) $w_{n} \subseteq w_{n+1}$

(v) $y_{n} \leq x_{n}, y_{n} \in B^{\prime}$.

(vi) for no $z \in\left\langle\cup_{\zeta<\xi} B_{[\zeta]} \cup\left\{a_{\alpha} \in w_{n}\right\}_{B_{0}^{c}}\right.$ is $\cap x_{n}=y_{n}$.

For $n=01 \notin F i\left(B^{\prime}\right)$

For every $n$ let $w_{n}$ be a finite subset of $\{\gamma: \zeta(\gamma)=\xi\}$ extending $\cup_{\ell<n} w_{\ell}$, such that for every $\ell<n, x_{\ell}, y_{\ell} \in\left\langle\cup_{\zeta<\xi} B_{[\zeta]} \cup\left\{a_{\alpha}: \alpha \in w_{n}\right\}\right\rangle_{B_{0}^{c}}$. Then as $1-\cup_{\ell<n} x_{i} \notin F i\left(B^{\prime}\right)$, and as $B^{\prime}$ is $\aleph_{1}$-compact, there is $x_{n} \leq 1-\cup_{i<n} x_{i}, x_{n} \in$ $B^{\prime}, 1-\cup_{\ell \leq n} x_{i} \notin F i\left(B^{\prime}\right)$ and $x_{n} \notin F i\left(B^{\prime}\right)$. Now as (**) fails $w_{n}, x_{n}$ does not satisfy the requirements on $w, x^{*}$ in $(* *)$, so there is $y_{n} \in B^{\prime}, y_{n} \leq x_{n}$ such that for no $z \in\left\langle\cup_{\zeta<\xi} B_{[\zeta]} \cup\left\{a_{\alpha}: \alpha \in w_{n}\right\}\right\rangle_{B_{0}^{c}}$. By 3.8 we can easily get a contradicition to (vi). So $(* *)$ holds.

Let $t_{0}, \ldots, t_{m} \in B_{[\xi]}$ be such that $\cup_{\ell=1}^{m} t_{\ell}=1$ and $(\forall \ell \leq m)(\forall \alpha \in w)\left[t_{\ell} \leq\right.$ $\left.a_{\alpha} \vee t-\ell \cap a_{\alpha}=0\right]$. There is an $\ell \leq m$ such that $\left\{u \cap t_{\ell}: y \leq x^{*}\right.$ and $\left.y \in B^{\prime}\right\}$ is finite. It is clear (by Part II) that $B^{\prime \prime}=\left\langle b^{\prime}, t_{\ell}\right\rangle_{B_{0}^{c}}$ is $\aleph_{1}$-compact: also $\left.\left.X^{*} \cap t_{\ell} \in B^{\prime \prime}-F i\right) B^{\prime \prime}\right)$. Now if $y \in B^{\prime \prime}, y \leq x^{*} \cap t_{\ell}$ then for some $y^{\prime} \in B^{\prime}, y=y^{\prime} \cap t_{\ell}$ and w.l.o.g. $y^{\prime} \leq x^{*}$, so for some $z \in\left\langle\cup_{\zeta<\xi} B_{[\zeta]} \cup\left\{a_{\alpha}\right.\right.$ : $\alpha \in w\}\rangle_{B_{0}^{c}} z \cap x^{*}=y^{\prime}$ hence $z \cap\left(x^{*} \cap t_{\ell}\right)=y$, and by the choice of $t_{\ell}$, for some $z^{\prime} \in \cup_{\zeta<\xi} B_{[\zeta]}$, the equation $z^{\prime} \cap\left(x^{*} \cap t_{\ell}\right)=z \cap\left(x^{*} \cap t_{\ell}\right)=y$ holds.

So $B^{\prime \prime}, x * * \stackrel{\text { def }}{=} x^{*} \cap t-\ell$ satisfy requirements in $(* *)$. Now we use (c) of part I. As $c f(\xi)=\aleph_{0}$, let $\xi=\cup_{n<\omega} \zeta_{n}$, and we define by induction on $n<\omega, x_{n}, y_{n}$ such that:

(i) $x_{n} \in B^{\prime \prime}, x_{n} \leq x^{* *}$

(ii) $x^{* *}-\cup_{\ell<n} x_{i} \notin F i\left(B^{\prime \prime}\right)$

(iii) $y_{n} \in B^{\prime}, y_{n} \leq x_{n}$

(iv) for no $z \in B_{\left[\zeta_{n}\right]}, z \cap x_{n}=y_{n}$.

As $B$ " is $\aleph_{1}$-compact, for some $x^{*} \in B^{\prime \prime}, z^{*} \cap x_{n}=y_{n}$ for each $n$.

Now as $B^{\prime \prime}, x^{* *}$ satisfy (**), for some $z^{* *} \in \cup_{\zeta<\xi} B_{[\zeta]} z^{*} \cap x^{* *}=z^{* *} \cap x^{* *}$. So for some $n z^{* *} \in B_{\left[\zeta_{n}\right]}$, contradicting (iv) above. Thus we have finished the proof of 3.10 .

3.11 Claim. $B_{\alpha^{*}}$ is endo-rigid.

Proof Suppose $h$ is a counterexample, i.e. $h$ is an endomorphism of $B_{\alpha^{*}}$ but $B_{\alpha^{*}} / \operatorname{Ex} \operatorname{Ker}(h)$ is infinite, and we shall get a contradiction.

Clearly if for some $\alpha, N^{\alpha}=\left(B^{*}, h\right) h$ maps $B^{*} \cap B_{\alpha^{*}}$, into itself and $\alpha \in J$ 
omits $p_{\alpha}$ ). So we shall try to find such $\alpha$ which satisfies the requirements in Stage B for belonging to $J$. We assume $N^{\alpha}=\left(\left|N^{\alpha}\right|, h_{\alpha}\right),\left|N_{\alpha}\right| \subseteq B_{\alpha}, h_{\alpha}=$ $h \uparrow N^{\alpha}, h_{\alpha}$ maps $N^{\alpha} \cap B_{\alpha}$ onto itself, and $N_{0}^{\alpha}$ contains some elements we need and somewhat more (see latter). As $W$ is a barrier this is posible. We then will choose $\eta_{\alpha}$, an $\omega$-branch of $f^{\alpha}$, distinct from $\eta_{\beta}$ for $\beta<\alpha$ [if $\beta+2^{\aleph_{0}} \leq \alpha$ this follows, the rest exclude $<2^{\aleph_{0}}$ branches of $f^{\alpha}$ but there are $2^{\aleph_{0}}$ such branches], a maximal antichain $\left\langle d_{n}: n<\omega\right\rangle$ of $B_{\alpha}, d_{n} \in N_{0}^{\alpha}$, and $\tau_{n} \in N^{\alpha}$ in $\left\langle x_{\nu}: \eta \alpha \uparrow n<\nu \in T\right\rangle_{B_{)}^{c}}$, and let $b_{n}=h\left(d_{n}\right), c_{n}=$ $h\left(d_{n} \cap \tau_{n}\right), p_{\alpha}=\left\{x \cap b_{n}=c_{n}: n<\omega\right\}$, and $a_{\alpha}=\cup_{n<\omega}\left(d_{n} \cap \tau_{n}\right) \in B_{0}^{c}$. All should have superscript $\bar{d}, \bar{\tau}$ (where $\bar{d}=\langle: n<\omega\rangle, \bar{\tau}=\left\langle\tau_{n}: n<\omega\right\rangle$ ) but we usually omit them or write $a_{\alpha}[\bar{\tau}, \bar{d}], p_{\alpha}[\bar{\tau} \bar{d}]$ etc.

The choice of $\bar{d}, \bar{\tau}$ ( and $\eta_{\alpha}$ which is determined by $\bar{\tau}$ ) is done by listing the demands on them (see Stage B) and showing a solution a solution exists. The only problematic one is (a) (omitting $p_{\beta}$ for $\beta \leq \alpha$ ) and we partition it to three cases:

(I) $\zeta(\beta)<\zeta(\alpha)$ or $\zeta(\beta)=\zeta(\alpha), \beta+2^{\aleph_{0}} \leq \alpha$,

(II) $\zeta(\beta)=\zeta(\alpha), \beta<\alpha<\beta+2^{\aleph_{0}}$.

(III) $\beta=\alpha$

We shall prove that every $\bar{\tau}, \bar{d}$ are O.K. for (I), that for any family $\left\{\left(\bar{d}^{i}, \eta^{i}, \bar{\tau}^{i}\right): i<2^{\aleph_{0}}\right\}$ ( $\eta$ a branch of $f^{\alpha}$, etc.) with pairwise distinct $\eta^{i}$ 's, all except $2^{\aleph_{0}}$ many are O.K. for instance of (II), and that there is a family of $2^{\aleph_{0}}$ triples $(\bar{d}, \bar{\eta}, \bar{\tau})$ satisfying (III) with pairwise distinct $\eta^{i}$ 's. This clearly suffices.

Case I: $\zeta(\beta)<\zeta(\alpha)$ or $\left(\zeta(\beta)=\zeta(\alpha), \beta+2^{\aleph_{0}} \leq \alpha\right.$

Suppose some $x \in\left\langle_{\alpha}, a_{\alpha}[\bar{\tau}, \bar{d}]\right\rangle_{B_{0}^{c}}$ realizes $p_{\beta}$. Clearly there is a partition $\left\langle e_{\ell}: \ell<4\right\rangle$ of 1 (in $\beta_{\alpha}$ ) such that $x=e_{0} \cup\left(e_{1} \cap a_{\alpha}[\bar{\tau}, \bar{d}]\right) \cup\left(e_{2}-a_{\alpha}[\bar{\tau}, \bar{d}]\right)$. Choose $\xi<\zeta(\alpha)$ large enough and finite $w \subseteq \alpha$ so that $[\zeta(\beta)<\zeta(\alpha) \Rightarrow$ $\zeta(\beta)<\xi], d_{n}, h_{\alpha}\left(d_{n}\right) b_{n}^{\beta}$, are based on $\left\{x_{\nu}: \nu \in{ }^{\omega>} \xi\right\}$ (for $\eta<\omega$ ) and $c_{\ell}^{\beta}(\ell<\omega), e_{0}, e_{1}, e_{2}, e_{3}$ are based on $J=\left\{\nu \in T: \eta_{\alpha}\lceil k \not L \nu\}\right.$, where $k<\omega$ also satisfies such that $\eta_{\alpha}(k)>\xi, \eta_{\alpha} k \notin N_{\beta}$.

We claim:

$(*)$ there is $m<\omega$ such that $b_{m}^{\beta} \cap\left(e_{1} \cup e_{2}\right)-\cup_{n \leq k} d_{n} \neq 0$

For suppose $\left(^{*}\right)$ fail, then as $a_{\alpha}[\bar{\tau}, \bar{d}] \cap\left(\cup_{n \leq k} d_{n}\right) \in B_{\alpha}$, w.l.o.g. $(e-1 \cup$ $\left.e_{2}\right) \cap \cup_{n \leq k} d_{n}=0$ (otherwise let

$$
\begin{aligned}
& e_{0}^{\prime}=e_{0} \cup\left(e_{1} \cap a_{\alpha}[\bar{\tau}, \bar{d}] \cap \cup_{n \leq k} d_{n}\right) \cup\left(e-2 \cap \cup_{n \leq k} d_{n}-a_{\alpha}[\bar{\tau}, \bar{d}]\right) \\
& e_{1}^{\prime}=e_{1}-\cup_{n \leq k} d_{n}, \\
& \left.e_{2}^{\prime}=e_{2}-\cup_{n \leq k} d_{n}\right) .
\end{aligned}
$$

So for every $M<W, b_{M}^{\beta} \cap\left(e_{1} \cup e_{2}\right)=0$.

So if $x$ realizes $p_{\beta}$ then so does $e_{0}$, but $e_{0} \in B_{\alpha}$ contradicting an induction hypothesis. So $(*)$ holds.

Now as $\left\langle d_{n}: n<\omega\right\rangle$ is a maximal antichain in $B_{\alpha}$, for some $\ell<\omega, d_{\ell} \cap$ $\left(b_{m}^{\beta} \cap\left(e_{1} \cup e_{2}-\cup_{n \leq k} d_{n}\right)\right) \neq 0$. Necessarily $\ell>k$. So for some $\epsilon \in\{1,2\}, d_{\ell} \cap$ $b_{m}^{\beta} \cap e_{\epsilon} \neq 0$. As $x$ realizes $p_{\beta}, x \cap\left(d_{\ell} \cap b_{m}^{\beta} \cap e_{\epsilon}\right)=d-\ell \cap c_{n}^{\beta} \cap e_{\epsilon}$ which is based on $J$. But we know that $x \cap\left(d_{\ell} \cap b_{m}^{\beta} \cap e_{\epsilon}\right)$ is $d_{\ell} \cap b_{m}^{\beta} \cap e_{1} \cap a_{\alpha}[\bar{\tau}, \bar{d}]=d_{\ell} \cap b_{m}^{\beta} \cap e_{1} \cap \tau_{\ell}$ (if $\epsilon=1)$ or $d_{\ell} \cap b_{m}^{\beta} \cap e_{2} \cap\left(1-a_{\alpha}([\bar{\tau}, \bar{d}])=d_{\ell} \cap b_{m}^{\beta} \cap e_{2} \cap 1-\tau_{\ell}\right.$ (if $\left.\epsilon=2\right)$. 
As $d_{\ell} \cap b_{m}^{\beta} \cap e-z \neq 0$ is based on $J, \ell>k, \eta_{\alpha}(k)>\xi, \tau_{\ell}$ is free over $J$, (see Fact(2)). Necessarily $x \cap\left(d_{\ell} \cap b_{m}^{\beta} \cap e_{z}\right)$ is not based on $J$, contradiction.

Case II: $\beta<\alpha<\beta+2^{\aleph_{0}}$

We shall prove that if $\eta^{\ell}, \bar{\tau}^{\ell}$ are appropiate (for $\left.\ell=1,2\right)$ and $\eta^{1} \neq \eta^{2}$ then $p_{\beta}$ cannot be realized in both $\left\langle B_{\alpha}, a\left[\bar{\tau}_{\ell}, \bar{d}\right]\right\rangle_{B_{0}^{c}}$. (So as $\beta<\alpha<\beta+2^{\aleph_{0}}$, there are less then $2^{\aleph_{0}}$ non appropiate $\left.\bar{\eta}^{?}, \bar{\tau}^{1}\right)$.

As there is a perfect set of appropiate $\eta$ 's it will suffice to prove that for each $\omega$-branch $\eta$ of $\operatorname{Rang}\left(f^{\alpha}\right)$ for some appropiate $\tau_{1}\left\langle B_{\alpha}, a^{\bar{\tau}}\right\rangle_{B_{0}^{c}}$ omits $p_{\alpha}=p_{\alpha}[\bar{\tau}, \bar{d}]$ which will be done in Case III.

Note that $I_{\beta}^{\alpha}=\left\{e \in B_{\alpha}\right.$ : for some $x \leq e$ for every $\left.n x \cap b_{\beta}^{n} \cap e=c_{\beta}^{n} \cap e\right\}$ is an ideal.

The details are easy.

Case III: $\beta=\alpha$

This case is splitted into several subcases. Let $\eta_{\alpha}$ be any $\omega$-branch of $f^{\alpha}, \eta_{\alpha} \neq \eta_{\beta}$ whenever $\beta<\alpha<\beta+2^{\aleph_{0}}$. Let $I^{*}=\cup\left\{\underline{d}(h(x)) ; x \in B_{\alpha}\right\}$. We shall assume that $\left|I^{*}\right| \leq \aleph_{0} \Rightarrow I^{*} \subseteq N_{0}^{\alpha}$, so in this case $p_{\alpha}$ is omitted by $B_{\alpha+1}$ or $B_{\alpha^{*}}$ iff ?? omitted by $B_{\alpha}$ (by $3.7(1)$ ). As acomplishing this aim is easier we shall ?? this case (work as in III 4 and use quite arbitrary $p_{\beta}$ ).

Subcase III 1.: For some $\rho^{*} \in T$, and $a^{*} \in B_{\alpha}-\operatorname{Ex} \operatorname{Ker}^{*}(h)$ for every $\leq \rho \in T$ for some $\tau \in\left\langle x_{\eta}: \rho<\eta \in T\right\rangle_{B_{0}^{c}}, \tau a \cap a * \neq 0=h\left(\tau \cap a^{*}\right)$.

As we are interested not in $\left.f^{\alpha}, N^{\alpha}\right)$ itself, but in $h$, by using $G m^{\prime}(W)$, w.l.o.g. $\rho^{*} \in$ Range $\left(f^{\alpha}\right)$. By 3.10 (for rang $(h)$, which by assumption, is infinite) ?? easy manipulations (see 2.4 and [Sh 2]) there is maximal antichain ?? : $n<\omega\rangle$ of $B_{\alpha^{*}}$ such that for no $x \in B_{\alpha}, x \cap h\left(2_{2 n}=h\left(d_{2 n}\right)\right.$ and $\cap h\left(d_{2 n+1}\right)=O$ W.l.o.g. $\left\{d_{n}: n<\omega\right\} \subseteq N_{0}^{\alpha}$

It suffices to prove the conclusion for any $\omega$-branch $\eta_{\alpha}$ of $\operatorname{Range}\left(f^{\alpha}\right), \rho^{*}<$ $\eta_{\alpha} \notin\left\{\eta_{\beta}: \beta<\alpha\right\}$. We define by induction on $n, \tau_{n} \in N_{n}^{\alpha}<i n\left\langle x_{\eta}\right.$ : $\eta_{\alpha}\lceil n \leq \eta\rangle_{B_{0}^{c}}, \tau_{n} \neq 0,1$ and $h\left(\tau_{2 n}\right)=1, h\left(\tau_{2 n+1}\right)=0$. (possible by the assumption of subcase III 1), so we finish this subcase.

Subcase III 2. For some $a^{*} \in B_{\alpha},\left\{h(x)-a^{*}: x \in B_{\alpha}, x \leq a^{*}\right\}$ is infinite.

Clearly $B^{a}=\left\{h(x)-a^{*}: x \in B_{\alpha^{*}} x \leq a^{*}\right\} \cup\left\{1-\left(h(x)-a^{*}\right): b_{\alpha^{*}} x \leq a^{*}\right\}$ is a subalgebra of $B_{\alpha^{*}}$ (with $a^{*}$ an atom). By assumption (of ?? subcase) $B^{a}$ is infinite. So by 3.9 there are $e_{n} \in B^{a}$, pairwise disjoint, and (? $\left.x \in B_{a}\right) \bigwedge_{n}\left(x \geq e_{2 n} \wedge x \cap e_{2 n+1}=0\right)$. As $a^{*}$ is an atom of $B^{a}$ w.l.o.g. $\leq 1-a^{*}$, hence there is $d_{n} \leq a^{*}$ (in $B_{\alpha^{*}}$, such that $h\left(d_{n}\right)=e_{n}$. Clearly ?? $\left.-\cup_{\ell<n} d_{n}\right)=e_{n}-\cup_{\ell<n} e_{\ell}=e_{n}$, so w.l.o.g. the $D_{n}$ are pairwise disjoint. So by easy manipulation for some $\left\langle d_{n}: n<w\right\rangle$ the following holds:

(i) $d_{0}=1-a^{*}$

(ii) $\left\langle d_{n}: n\langle\omega\rangle\right.$ is a maximal antichain of $B_{\alpha^{*}}$.

(iii) for no $x \leq 1-a^{*} x \cap h\left(d_{2 n+2}\right)-a^{*}, x \cap h\left(d_{2 n+1}\right)-a^{*}=0$

We can assume that $d_{n}, h\left(d_{n}\right) \in N_{0}^{\alpha}$.

Let $\bar{\tau}=\left\langle\tau_{n}^{0}: n<\omega\right\rangle$ be a suitable suquence, (for our $\eta_{\alpha}$ ) then so are $\bar{\tau}^{\ell}=\left\langle\bar{\tau}^{\ell}, \omega\right\rangle$ for $\ell<4$ where: 
$\tau_{2 n}^{1}=1-\tau_{2 n}^{0}, \tau_{2 n+1}^{1}=\tau_{2 n+1}^{0}$
$\tau_{2 n}^{2}=\tau_{2 n}^{0}, \tau_{2 n+1}^{2}=1-\tau_{2 n+1}^{0}$
$\tau_{2 n}^{3}=1-\tau_{2 n}^{0}, \tau^{3} 2 n+1=1-\tau_{2 n+1}^{0}$

Suppose for each $\ell<4$, in $\left\langle_{\alpha}, a_{\alpha}\left[\bar{\tau}^{\ell}, \bar{d}\right]\right\rangle_{B_{0}^{c}}$ there is an element $y^{\ell}$ which satisfies $y^{\ell} \cap h\left(d^{\ell}\right)-a^{*}=h\left(\tau_{n}^{\ell} \cap d_{n}\right)-a^{*}$ for $1 \leq n<\omega$. W.l.o.g. $y^{\ell} \leq$ $1-a^{*}=d_{0}$ hence $y^{\ell} \in B_{\alpha}$. Now $\left(y^{0} \cup y^{1} \cap\left(y^{2} \cup y^{3}\right) \in B_{\alpha}\right.$ contradict (iii) above.

Subcase III 3. For some $a^{*} \in B_{\alpha}^{*}-E x \operatorname{Ker}^{*}(h)$, and $\rho^{*} \in T$, for every $\rho, \rho \leq \rho \in T$ there is $\tau \in\left\langle x_{\nu} ; \rho \leq \nu \in T\right\rangle_{B_{0}^{c}}$ such that $h\left(\tau \cap a^{*}\right) \cap a^{*}=\tau \cap a^{*}$

Clearly the function $h^{\prime}: b_{\alpha}^{*} \uparrow a^{*} \rightarrow B_{\alpha}^{*} \uparrow a^{*}$ defined by $h^{\prime}(x)=h(x) \cap a^{*}$ is an endomorphism; W.l.o.g. the assumption of subcase III 2 fails hence $\left\{(x)-a^{*}: x \leq a^{*}\right\}$ is finite, hence the range of $h^{\prime}$ is finite (as $a^{*} \notin$ $\operatorname{Ex} \operatorname{Ker}^{*}(h)$, so by 2.4 there is $x \leq a^{*}$ such that $h(x) \cap a^{*}-x \neq 0$; we know that $\underline{d}(x)$ is countable, hence for some $\rho^{* *}, \rho^{*} \leq \rho^{* *} \in T,\{\nu: \rho \leq \nu \in T\}$ is disjoint to $\left.\left(a^{*}\right) \cup \underline{d}(x)\right)$. Now by the hypothesis of subcase III 3 we can easily find $\tau_{n} \in\left\langle x_{\nu}: \rho^{* *} \leq \nu \in t\right\rangle_{B_{0}^{c}}$, with pairwise disjoint $\bar{d}\left(\tau_{n}\right)$ and $h\left(\tau_{n} \cap a^{*}\right) \cap a^{*}=\tau_{n} \cap a^{*}$. So

$h\left(\tau_{n}(\cap x) \cap\left(a^{*}-x\right)=h\left(\left(\tau_{n} \cap a^{*}\right) \cap x\right) \cap\left(a^{*}-x\right)=h\left(\tau_{n} \cap a^{*}\right) \cap h(x) \cap\right.$ $\left(a^{*}-x\right)=\left(h\left(\tau_{n} \cap a^{*}\right) \cap a^{*}\right) \cap h(x) \cap\left(a^{*}-x\right)=\left(\tau_{n} \cap a^{*}\right) \cap h(x) \cap\left(a^{*}-x\right)=$ $\tau_{n} \cap h(x) \cap\left(a^{*}-x\right)=\tau_{n} \cap\left(h(x) \cap a^{*}-x\right)$

It is $\neq 0$ as $\left.\left.\bar{d}\left(\tau_{n}\right) \cap \bar{d}(x) \cup \bar{d}(h(x)) \cup \bar{d}\left(a^{*}\right)\right)=\phi\right)$ and $h(x) \cap a^{*}-x \neq$ $\left.0, \tau_{n} \neq 0\right]$, and for different $n$ we get different values. So $\left\{h(y \cap x) \cap\left(a^{*}-x\right)\right.$ : $\left.x \in B_{\alpha}^{\prime}\right\}$, is finite. Hence $\left\{(y \cap x)-x ; y \in B_{\alpha^{\prime}}\right.$ is infinite. Leading to the assumption of subcase III 2(with $x$ here for $a^{*}$ there).

Subcase III. 4 For some $\rho^{*} \in T$, and $a^{*} \in B_{\alpha^{*}} \operatorname{Ex} \operatorname{Ker}^{*}(h)$ for every $\tau \in\left\langle{ }_{x} \nu: \rho^{*} \not \leq \nu \in T\right\rangle_{B_{0}} h\left(\tau \cap a^{*}\right) \cap a^{*}$ is based on $\left\{\nu: \rho^{*} \not \leq \nu \in T\right\}$.

W.l.o.g. the hypothesis of subcase III 1 fails hence $\left\{h\left(\tau \cap a^{*}\right): \tau \in\left\langle x_{\nu}\right.\right.$ : $\left.\left.\rho^{*} \leq \nu \in T\right\rangle_{B_{0}^{c}}\right\}$ is infinite. As also w.l.o.g. the hypothesis of subcase III 2 fails we get $\left\{\left(\tau \cap a^{*}\right) \cap a^{*}: \tau \in\left\langle x_{\nu}: \rho^{*} \leq \nu \in T\right\rangle_{B_{0}^{c}}\right\}$ is infinite. So by 3.9 we can find $d_{n} \in\left\langle x_{\nu}: \rho^{*} \leq \nu \in T\right\rangle_{B_{0}^{c}}$ such that $\left.d_{n}: n<\omega\right\rangle$ is a maximal antichain in $B_{0}^{c}$, and there is no $x \in B_{\alpha^{*}}, z \cap h\left(d_{2 n}=h\left(d_{2 n}\right)+h\left(d_{2 n}\right), x \cap\right.$ $h\left(d_{2 n+1}\right)=0$, and $d_{0}=1-a^{*}$.

As before we can assume $\rho^{*} \in \operatorname{Rang}\left(f^{\alpha}\right)$ and $d_{n} \in N_{0}^{\alpha}$ for $n<\omega$. We suppose $\eta_{\alpha} \notin\left\{\eta_{\beta}: \beta<\alpha\right\}$ is an $\omega$-branch of $f^{\alpha}, \rho^{*} \leq \eta_{\alpha}$.

For any suitable $\tau$ if $y[\bar{\tau}, \bar{d}] \in\left\langle B_{\alpha}, a_{\alpha}[\bar{\tau}, \bar{d}]\right\rangle_{B_{0}^{c}}$ satisfies $\tau_{n} \in\left\langle x_{\nu}: \rho^{*} \leq\right.$ $\nu \in T\rangle_{B_{0}^{c}}$ and $y[\bar{\tau}, \bar{d}] \cap h\left(d_{n}\right)$, (for every $n$ ) then by 3.3 we easily get $y[\bar{\tau}, \bar{d}] \in$ $B_{\alpha}$, and then get contradiction by trying four $\tau$ 's, as in subcase III 2 .

Subcase III. 5. There are $\rho^{*} \in T$ and an atomless countable subalgebra $Y \subseteq B_{\alpha}^{*}$ and pairwise disjoint $c_{\ell} \in Y(\ell<\omega)$ such that for every $\ell$ and $\rho_{\ell} \in\left\{\rho: \rho^{*} \leq \rho \in T\right\}$ for some $\tau_{\ell} \in\left\langle x_{\nu}: \rho_{\ell} \leq \nu \in T\right\rangle_{B_{0}^{c}}$, the following holds: for no $x \in B_{0}^{c}$ is $\bar{d}(x) \subseteq\left\{\nu: \rho_{\ell} \not \leq \nu \in T\right\}$ and $z \cap h\left(c_{\ell}\right) \cap c_{\ell}-\tau_{\ell}=$ $h\left(c_{\ell} \cap \tau_{\ell}\right) \cap c_{\ell}-\tau_{\ell}$.

Let $\left\langle D_{n}: n<\omega\right\rangle$ be a maximal antichain of $B_{\alpha^{*}}$ such that $d_{2 n}=c_{2 n}$

So w.l.o.g. $Y \cup\left\{d_{n}: n<\omega\right\} \subseteq N_{0}^{\alpha}, \rho^{*} \in \operatorname{Rang}\left(f^{\alpha}\right)$ (using $\operatorname{Gm}^{\prime}(W)$ ), 
We can now choose by induction on $n, t_{n} \in N_{n}^{\alpha}$,

$$
\tau_{n} \in\left\langle x-\nu: \eta_{\alpha}\lceil n \leq \nu \in T\rangle_{B_{0}^{c}}\right.
$$

such that

$\left(^{*}\right)$ (a) for even $n$, for no $x \in B_{0}^{c}$ based on $\left\{\nu: \eta_{\alpha}\lceil n \not \leq \nu \in t\}\right.$ is $x \cap h\left(d_{n}\right) \cap d_{n}-\tau_{n}=h\left(d_{n} \cap \tau_{n}\right) \cap d_{n}=\tau_{n}$.

Why is this sufficient? We let $\bar{d}=\left\langle d_{n}: n\langle\omega\rangle\right.$ and $\bar{\tau}=\left\langle\tau_{n}: n\langle\omega\rangle\right.$. So assume some $y[\bar{\tau}, \bar{d}] \in\left\langle B_{\alpha}, a_{\alpha}[\bar{\tau}, \bar{d}]\right\rangle_{B_{0}^{c}}$ realizes $p_{\alpha}[\bar{\tau}, \bar{d}]$, i.e. satisfies

$y[\bar{\tau}, \bar{d}] \cap h\left(d_{n}\right)=h\left(d_{n} \cap \tau_{n}\right)$ for every $n$. As $y[\bar{\tau}, \bar{d}] \in\left\langle B_{\alpha}, a_{\alpha}[\bar{\tau}, \bar{d}]\right\rangle_{B_{0}^{c}}$ for $y[\bar{\tau}, \bar{d}]=e_{0}[\bar{\tau}, \bar{d}] \cup\left(e_{1}[\bar{\tau}, \bar{d}] \cap a_{\alpha}[\bar{\tau}, \bar{d}]\right) \cup\left(e_{2}[\bar{\tau}, \bar{d}]-a_{\alpha}[\bar{\tau}, \bar{d}]\right)$.

For some $m(*), \omega, \underline{d}\left(e_{0}[\bar{\tau}, \bar{d}]\right) \cup \underline{d}\left(e_{1}[\bar{\tau}, \bar{d}] \cup \underline{d}\left(e_{2}[\bar{\tau}, \bar{d}]\right)\right.$ is disjoint to $\{\nu$ : $\eta_{\alpha}\lceil m(*) \leq \nu \in T\}($ see $3.3(2))$.

Now we compute for $n$ even $>m(*)$ :

$z \stackrel{\text { def }}{=} h\left(d_{n} \cap \tau_{n}\right) \cap d_{n}-\tau_{n}=$

$=y[\bar{\tau}, \bar{d}] \cap h\left(d_{n}\right) \cap d_{n}-\tau_{n}$ by the choice of $\left.y[\bar{\tau}, \bar{d}]\right)$

$=\left(e_{0}[\bar{\tau}, \bar{d}] \cup\left(e_{1}[\bar{\tau} \bar{d}] \cap a_{\alpha}[\bar{\tau}, \bar{d}]\right) \cup\left(e_{2}[\bar{\tau}, \bar{d}]-a[\bar{\tau}, \bar{d}]\right)\right) \cap h\left(d_{n}\right) \cap d_{n}-\tau_{n}=$

$=\left(e_{0}[\bar{\tau}, \bar{d}] \cap h\left(d_{n}\right) \cap d_{n}-\tau_{n}\right) \cup\left(\left(e-1[\bar{\tau}, \bar{d}] \cap a_{\alpha}[\bar{\tau}, \bar{d}]\right) \cap h\left(d_{n}\right) \cap d_{n}-\tau_{n}\right) \cup$ $\cup\left(\left(e_{2}[\bar{\tau}, \bar{d}]-a_{\alpha}[\bar{\tau}, \bar{d}]\right) \cap h\left(d_{n}\right) \cap d_{n}-\tau_{n}\right)$

But $a_{\alpha}[\bar{\tau}, \bar{d}] \cap d_{n}=\tau_{n} \cap d_{n}$ hence

$$
\begin{gathered}
\left(e_{1}[\bar{\tau}, \bar{d}] \cap a_{\alpha}[\bar{\tau}, \bar{d}]\right) \cap d_{n}=\left(e _ { 1 } \left[\bar{\tau}, \bar{d} \cap \tau_{n} \cap d_{n}\right.\right. \\
\left(e_{2}[\bar{\tau}, \bar{d}]-a_{\alpha}[\bar{\tau}, \bar{d}]\right) \cap d_{n}=\left(e_{2}[\bar{\tau}, \bar{d}]-\tau_{n}\right) \cap d_{n}
\end{gathered}
$$

Hence

$\left.z=\left(e_{0}[\bar{\tau}, \bar{d}] \cap h\left(d_{n}\right) \cap d_{n}-\tau_{n}\right) \cup\left(e_{1}[\bar{\tau}, \bar{d}] \cap \tau_{n}\right) \cap h\left(d_{n}\right) \cap d_{n}-\tau_{n}\right) \cup$ $\left(\left(e_{2}[\bar{\tau}, \bar{d}]-\tau_{n} \cap h\left(d_{n}\right) \cap d_{n}-\tau_{n}\right)\right.$

But the second term is zero and in the first $-\tau_{n}$ is redundant, so

$\left.z=\left(e_{0}[\bar{\tau}, \bar{d}] \cap h\left(d_{n}\right) \cap d_{n}-\tau_{n}\right) \cup e_{2} \cap h\left(d_{n}\right) \cap d_{n}-\tau_{n}\right)=$

$=\left(e_{0}[\bar{\tau}, \bar{d}] \cup e_{2}[\bar{\tau}, \bar{d}]\right) \cap h\left(d_{n}\right) \cap-\tau_{n}$

We can conclude

$\left(e_{0}[\bar{\tau}, \bar{d}] \cup e-2[\bar{\tau}, \bar{d}]\right) \cap h\left(d_{n}\right) \cap d_{n}-\tau_{n}=h\left(d_{n} \cap \tau_{n}\right) \cap d_{n}-t_{n}$

contradicting the choice of $\tau_{n}$.

To finish Case III (hence the proof of 3(10) we need only

Why the five subcases exhaust all posibilities?

Suppose none of III 1-5 occurrs. By not subcase III 1 for some $\rho^{0} \in T$,

(a) $h(\tau) \neq 0$ for every $\tau \in\left\langle x_{\eta}: \rho^{0} J \leq \eta \in T\right\rangle_{B_{0}^{c}}$

Let $Y$ be the $\left\langle x_{\rho^{0}<i>}:<\omega\right\rangle_{B_{0}^{c}}$. As $Y$ is countable, for some $i(*)<\lambda,\left\{\nu: \rho^{0}<i(*)>\leq \nu \in T\right\}$ is disjoint to $\cup\{\underline{d}(y) \cup \underline{d}(h(y))$ : $y \in Y\}$. As " not subcase III 5" for some $\rho^{1}, \rho^{0-}\langle i(*)\rangle \leq \rho_{1} \in T$, and

(b) there are no pairwise disjoint non zero $c_{\ell} \in Y(\ell<\omega)$, such that for every $\rho_{\ell}^{1}, \rho^{1}<\rho_{\ell}^{1} \in T$ for some $\tau_{\ell} \in\left\langle x_{\nu}: \rho_{\ell}^{1} \leq \nu \in T\right\rangle_{B_{0}^{c}}$, the following holds:

(*) for no $x \in B_{0}^{c}, \bar{d}(x) \subseteq\left\{\nu: \rho_{\ell}^{1} \not \leq \nu \in T\right\}$ and $x \cap h\left(c_{\ell}\right) \cap c_{\ell}-\tau_{\ell}=$ 
Clearly

(c) $\cup\{\underline{d}(y) \cup \underline{d}(h(y)): Y \in Y\}$ is disjoint to $\left\{\nu: \rho^{1} \leq \in T\right\}$

Let $Z+\left\{c \in Y\right.$ : for some $\rho_{c}^{1}, \rho^{1} \leq \rho_{c}^{1} \in T$ for no $\tau \in\left\langle x_{\nu}: \rho^{1} \leq\right.$ $\nu \in T\rangle_{B_{0}^{c}}$ does $(*)$ of (b) hold with $c, \tau$ instead $\left.c_{\ell}, \tau_{\ell}\right\}$

By (b) among any $\aleph_{0}$ pairwise disjoint members of $Y$, al least one belong to $Z$.

It is quite easy to define $y_{n} \in Z\left((n<\omega)\right.$ such that $\left[y_{n} \in\right.$ $\left.\operatorname{Ex} \operatorname{Ker}^{*}(h) \Rightarrow y_{n} \in \operatorname{Ex} \operatorname{Ker}(h)\right],\left[m<n \Rightarrow y_{n} \cap y_{m}=0\right]$, and for every $y \in Y-\{0\}$ for some $\left.n, y \cap\left(\cup_{\ell<n} y\right) \ell\right) \neq 0$ or $y_{n} \leq y$. So (by the choice of $Y$ ) $\left\langle y_{n}: n<\omega\right\rangle$ is maximal antichain of $B_{0}^{c}$. We shall show $y_{n} \in \operatorname{Ex} \operatorname{Ker}(h)$; fix $n$ for a while, and suppose $y_{n} \notin \operatorname{Ex} \operatorname{Ker}(h)$, and let $\rho_{n}^{1}, \rho_{n}^{1} \leq \rho_{n}^{1} \in T$ be such that for no $\tau \in\left\langle x_{\nu}: \rho_{n}^{1} \leq \nu \in T\right\rangle_{B_{0}^{c}}$ does (*) of (b) hold.

Now for each $\tau \in\left\langle x_{\nu}: \rho_{n}^{1} \leq \nu \in T\right\rangle_{B_{0}^{c}}$ as $y_{n} \in Z$, clearly [as (*) of (b) fail for $y_{n}, \tau\left(\right.$ and $\left.\left.\rho_{n}^{1}\right)\right]$ for some $x_{1} \in B_{0}^{c}, \bar{d}\left(x_{1}\right) \subseteq\left\{\nu: \rho_{n}^{1} \not \leq \nu \in\right.$ $T\}$ and $x_{1} \cap h\left(y_{n}\right) \cap y_{n}-\tau=h\left(y_{n} \cap \tau\right) \cap y_{n}-\tau$. Applying the failure of $\left(^{*}\right)$ of (b) for $y_{n}, 1-\tau, \rho_{n}^{1}$ we get $x_{2} \in B_{c}^{0}, \bar{d}\left(x_{2}\right) \subseteq\left\{\nu: r h o_{n}^{1} \not \leq \nu \in t\right\}$ and $x_{2} \cap h\left(y_{n}\right) \cap y_{n}-(1-\tau)=h\left(y_{n} \cap(1-\tau)\right) \cap y_{n}(1-\tau)$; note that $h\left(y_{n} \cap \tau\right) \leq h\left(y_{n}\right)$, and $h\left(y_{n} \cap(1-\tau)\right)=h\left(y_{n}\right)-h\left(y_{n} \cap \tau\right)$. By these equations and as $y_{n} h\left(y_{n}\right), x_{1}, x_{2}$ are based on $\left\{\nu: \rho_{n}^{1} \nless\right.$ $\nu \in T\}$ (by (c) and their choice resp.) clearly for some partition of $1, e_{0}^{\tau}, e_{1}^{\tau}, e_{2}^{\tau}, e_{3}^{\tau}, \in B_{0}^{c}$, based on $\left\{\nu: \rho^{1} \not \leq \nu \in T\right\}$ :

(i) $h\left(\tau \cap y_{n}\right) \cap y_{n}=e_{0}^{\tau} \cup\left(e_{1}^{\tau} \cap \tau\right) \cup\left(e_{2}^{\tau}-\tau\right)$.

Now for any $\tau, \sigma \in\left\langle x_{n} u: \rho_{n}^{1} \leq \in T\right\rangle$, easily (as $h$ is an endomorphism):

(ii) $h\left(\left(\tau \cup \sigma \cap y_{n}\right) \cap y_{n}=\left(h\left(\tau \cap y_{n}\right) \cap y_{n}\right) \cap\left(h\left(\sigma \cap y_{n}\right) \cap y_{n}\right)\right.$.

(iii) $h\left(\left(\tau \cup \sigma \cap y_{n}\right) \cap y_{n}=\left(h\left(\tau \cap y_{n}\right) \cap y_{n}\right) \cup\left(h\left(\sigma \cap y_{n}\right) \cap y_{n}\right)\right.$ i

We can apply (i) to $\tau, \sigma$ and also to $\tau \cup \sigma$, and substitute in (ii) (iii).

We get that

$(\alpha) e_{2}^{\tau} \cap e_{2}^{\sigma}=0$ if $\left.\left.\bar{d}(\tau) \cap \overline{(} \sigma\right)=\right), \tau, \sigma \in\left\langle x_{\nu} ; \rho^{1} \leq \in T\right\rangle_{b_{0}^{c}}$ (otherwise substitute (i) in (ii) and intersect with $e_{2}^{\tau} \cap e_{2}^{\sigma}$ ) and get $\left(h\left((\tau \cap \sigma) \cap y_{m}\right) \cap e_{2}^{\tau} \cap e_{2}^{\sigma}\right)=\left(e_{2}^{\tau}-\tau\right) \cap\left(e_{2}^{\sigma}-\sigma\right)=e_{2}^{\tau} \cap \tau_{2}^{\sigma} \cap(\tau \cup \sigma)$, and $\left(h\left((\tau \cap \sigma) \cap y_{n}\right) \cap y_{n}\right) \cap\left(e_{2}^{\tau} \cap e_{2}^{\sigma}\right) \notin\left\langle\left\{x: \bar{d}(x) \subseteq\left\{\nu: \rho_{m}^{1} \leq \nu \in\right.\right.\right.$ $T\} \cup(\tau \cap \sigma)\rangle_{B_{0}^{c}}$ contradiction to (i) for $\left.\sigma \cap \tau\right)$.

So let $\left\{\tau^{i}: i<\alpha\right\}$ be maximal such that $\bar{d}\left(\tau_{i}\right)$ are pairwise disjoint $e_{2}^{\tau^{i}} \neq 0$, and $\tau^{i} \in\left\langle x_{\nu}: \rho_{n}^{1} \leq \nu \in T\right\rangle_{B_{0}^{c}}$, then $\alpha<\omega_{1}$, we can choose $\rho_{n}^{2}$ such that:

$\rho_{n}^{1} \leq \rho_{n}^{2} \in T$, and $\left[\tau \in\left\langle x_{\nu}: \rho_{n}^{2} \leq \nu \in \tau\right\rangle_{B_{0}^{c}} \Rightarrow e_{2}^{\tau}=0\right.$.

Next we can get

$(\beta) e^{\tau}-1 \cap e_{0}^{\sigma}=0$ (if $\underline{d}(\tau) \cap \underline{d}(\sigma)=$ ), and $\tau, \sigma \in\left\langle x_{\nu}: \rho_{n}^{2} \leq\right.$ $\left.\nu \in T\rangle_{B_{0}^{c}}\right)$

The proof is similar to that of $(\alpha)$, using $\tau \cap \sigma$

As $B_{0}^{c}$ satisfies the $\aleph_{1}$-c.c. we can find $\left\{\tau^{i}: i<\omega\right\} \subseteq\left\langle x_{\nu}\right.$ : $\left.\rho_{n}^{2} \leq \nu \in T\right\rangle_{B_{0}^{c}}$, such that (in $B_{0}^{c} e_{\ell}^{*} \stackrel{\text { def }}{=} \cup_{i<\omega} e_{\ell}^{\tau^{i}}=\cup\left\{e_{\ell}^{\tau}: \tau \in\right.$ $\left.\left\langle x_{\nu}: \rho_{n}^{2} \leq \nu \in T\right\rangle_{B_{0}^{c}}\right\}$ for $\ell=0,1$. We can find $\rho_{n}^{3}, \rho_{n}^{2} \leq \rho_{n}^{3} \in t$, such that $\cup_{\ell<\omega} \underline{d}\left(\tau^{i}\right)$ is disjoint to $\left\{\nu: \rho_{n}^{3} \leq \nu \in T\right\}$. So for every 
$\tau \in\left\langle x_{\nu} \rho_{n}^{3} \leq \nu \in T\right\rangle_{B_{0}^{c}}, e_{0}^{\tau} \leq e_{0}^{*}$ (by the choice of $e_{0}^{*}$ ), and $e_{0}^{\tau} \cap e_{1}^{\tau^{i}}=0$ for $i<\omega$ (by $(\beta))$ hence $e_{0}^{\tau} \cap e_{1}^{*}=0$, hence

$$
(\gamma) e_{0}^{\tau} \leq e_{0}^{*}-e_{1}^{*}
$$

Similarly

$$
(\delta) e_{1}^{\tau} \leq e_{1}^{*}-e_{0}^{*}
$$

Now we can prove that $e_{1}^{\tau}=e_{1}^{\sigma}$ when $\bar{d}(\tau) \cap \bar{d}(\sigma)=0, \tau, \sigma \in\left\langle x_{\nu}\right.$ : $\left.\rho_{n}^{3} \leq \nu \in T\right\rangle_{B_{0}^{c}}$, repeat the proof of $(\alpha)$ intersecting with $e_{1}^{\tau}=e_{1}^{\sigma}$ when $\tau, \sigma \in\left\langle x_{\nu}: \rho_{n}^{3} \leq \in T\right\rangle_{B_{0}^{c}}$. So let $e_{1} \in B_{\alpha^{*}}$ be the common value, so

$(*) h\left(\tau \cap y_{n}\right) \cap y_{n}=e_{0}^{\tau}\left(e_{1} \cap \tau\right)$ for $\tau \in\left\langle x_{\nu}: \rho_{n}^{3} \leq \in T\right\rangle_{B_{0}^{c}} ;$ and $e_{0}^{\tau} \leq y_{n}-e_{1}$,

Let $e_{o}=y_{n}=e_{1}$, so $y_{n}=e_{0} \cup e_{1}, e_{0} \cap e_{1}=0$.

So $e_{0}^{\tau} \leq e_{0}$ for every $\tau \in\left\langle x_{\nu}: \rho_{n}^{3} \leq \nu \in T\right\rangle_{B_{0}^{c}}$

As $y_{n} \notin E x \operatorname{Ker}^{*}(h)$, at least one of the elements, $e_{0}, e_{1}$ is not in $\operatorname{Ex} \operatorname{Ker}(h)_{i}$. As not subcase III 2, for $\ell=1,2$ the homomorphism $G_{\ell}$ from $B_{\alpha^{*}}\left\lceil e_{\ell}\right.$ to $B_{\alpha^{*}} \uparrow\left(1-e_{\ell}\right), g_{\ell}(x)=h(x)-e_{\ell}\left(\right.$ for $\left.x=e_{1}\right)$ has a finite range. Hence for some ideal $\mathcal{J}$ of $B_{0}^{c} y_{n} / \mathcal{J}$ is a finite union of atoms and

for every $\tau \in e\left\langle x_{\nu}: \rho_{n}^{3} \leq \nu \in T\right\rangle \cap \mathcal{J}$

for $\ell=0,1 h\left(\tau \cap y_{n}\right) \cap e_{\ell}=h\left(\tau \cap e_{\ell}\right) \cap e_{\ell}$

hence $h\left(\tau \cap e_{\ell}\right) \cap e_{\ell}=\left(e_{0}^{\tau} \cup\left(e_{1}^{\tau} \cap \tau\right)\right) \cap e_{\ell}$.

So (for $\tau \in\left\langle x_{\nu}: \rho_{n}^{3} \leq \nu \in T\right\rangle_{B_{0}^{c}} \cap \mathcal{J}$ :

$h\left(\tau \cap e_{0}\right) \cap e_{0}=e_{0}^{\tau}$

$h\left(\tau \cap e_{1}\right) \cap e_{1}=\tau \cap e_{1}$

If $e-1 \notin E x \operatorname{Ker}(h)$, we get contradicition to "not subcase 3" [use $\rho_{n}^{3}$ for $\rho^{*}$ there, now for any $\rho, p_{n}^{3} \leq \rho \in T$ choose pairwise disjoint $\tau_{\ell} \in\left\langle x_{\nu}: \rho \leq \in T\right\rangle_{B_{0}^{c}}$ for $\ell<\omega$ by the choice of $\mathcal{J}$ for at least one $\ell, \tau_{\ell} \in \mathcal{J}$, so $\tau_{\ell}$ is as requires there]. So assume $e_{0} \notin$ $\operatorname{Ker} \operatorname{Ker}^{*}(h)$ and get contradiction to "not subcase III 4" [for some $\left.\ell<m<\omega x_{\rho_{n}^{3 \imath}<\ell>}-x_{\rho_{n}^{3 \imath}<n>}\right)$ for $\rho^{*}, a^{*}$ with $\alpha$ large enough].

So for each $n, y_{n} \in \operatorname{Ex} \operatorname{Ker}(h)$, by their choice) so let $y_{n}=$ $y_{n}^{0} \cup y_{n}^{1}$ (both in $\left.B_{\alpha^{*}}\right), h\left(y_{n}^{0}\right)=0, h(x)=x$ for $x \leq y_{n}^{1}, x \in B_{\alpha^{*}}$. Let $I \subseteq T$ be a countable set such that $\underline{d}\left(y_{n}^{0}\right), \underline{d}\left(y_{n}^{1}\right) \subseteq I$, and for $x-\in B_{\alpha^{*}} \underline{d}\left(h\left(x-y_{n}\right) \cap y_{n}\right) \subseteq I$ (by "not subcase III 2", for each $n$ we have only finitely many elements of this form).

We can easily show that for every $x \in B_{\alpha^{*}}$, for some $\alpha \in B_{0}^{c}$ based on $I, h(x)-x=a-x$, [as $\left\langle y_{n}: n<\omega\right\rangle$ is a maximal antichain in $B_{\alpha^{*}}$, for this it suffices to show for every $n<\omega$ there is $a_{n} \in B_{\alpha^{*}}^{c}, a_{n} \leq y_{n}$ such that $(h(x)-x) \cap y_{n}=a_{n}-x$; but $(h(x)-x) \cap y_{n}$ is the union of $\left(h\left(x \cap\left(y_{n}\right)-x \cap y_{n}\right.\right.$ which is zeto as $\left(\forall_{z} \leq y_{n}\right) h(z) \leq z$ and of $\left(h\left(x-y_{n}\right)-x\right) \cap y_{n}$ which we know is bsed as wanted]. so $\left.h(x)=e_{0}^{x} \cup\left(e_{1}^{x} \cap x\right) \cup e_{2}^{x}-x\right)$ where each $e_{\ell}^{x}$ is based on $I,\left\langle e_{\ell}^{x}: \ell<4\right\rangle$ pairwise disjoint $e_{\ell}^{*} \in B_{0}^{c}$. As in the analysis above of $h\left(x \cap y_{n}\right) \cap y_{n}$, (possibly with increasing $I$ ) applied to $x \in B_{\alpha^{*}}$ with $\underline{d}(x) \cap I=0$, we get $e_{2}^{x}=0, e_{1}^{x}=e_{1}$. If $e_{1} \notin E x \operatorname{Ker}^{*}(h)$ we get contradiction to "not subcase III 3". So $1-e_{1} \notin \operatorname{Ex} \operatorname{Ker}^{*}(h)$ and apply "not subcase III 4".

So we finish the proof of 3.11 ; so $B_{\alpha^{*}}$ is endo-rigid. 
Lemma. $B_{\alpha^{*}}$ is indecomposable.

Proof Suppose $K_{0}, K_{1}$ are disjoint ideals of $B_{\alpha^{*}}$, each with no maximal members, which generate a maximal ideal of $B_{\alpha^{*}}$. For $\ell=1,2$ let $\left\{d_{n}^{\ell}: \ell<\right.$ $\omega\}$ be a maximal antichain $\subseteq K_{\ell}$ (they are countable as $B_{\alpha^{*}}$ satisfies the c.c.c., and may be chosen infinite as $K_{\ell} \neq\{0\}, b_{\alpha^{*}}$ is atomless). Let $K$ be the ideal $K_{0} \cup K_{1}$ generates.

Now, e.g. for some $\xi, \lambda,\left\{d_{n}^{\ell}: \ell<2, n, \omega\right\} \subseteq B_{\xi}$. Clearly $a_{<\xi>}=$ $b^{0} \cup b^{1}, b^{\ell} \in K_{\ell}$. Now $\operatorname{pr}_{\xi}\left(b^{\ell}\right) \in B_{[\xi]}$ and is disjoint to each $d_{n}^{1-\ell}: n<$ $\omega\}, \operatorname{pr}_{\xi}\left(b^{\ell}\right)$ is disjoint to every member of $K_{1-\ell}$. As $K-0 \cup K_{1}$ generate a maximal ideal, clearly $\operatorname{pr}_{\xi}\left(b^{\ell}\right) \in K_{\ell}$ [otherwise $\operatorname{pr}_{\xi}\left(b^{\ell}\right)=1-c^{1} \cup c^{2}$, for some $c^{1} \in K_{1}, c^{2} \in K_{2}$, and then $c^{1-\ell}$ is necessarily a maximal member of $K_{1-\ell}$, so $K_{1-\ell}$ is principal contradiction]. So $\operatorname{pr}_{\xi}\left(B^{0}\right) \cup p r_{\xi}\left(b^{2}\right)<1$ but $1=p r_{\xi}\left(a_{<\xi>}=\cup_{\ell=0}^{2} p r_{\xi}\left(b^{\ell}\right)\right.$ contrdiction.

3.13 Theorem. In 3.1 we can get $2 \lambda^{\aleph_{0}}$ such Boolean Algebras such that any homomorphism from one to the other has a finite range.

PROOF Left to the reader (see [Sh 4.3]). 


\section{REFERENCES}

[CGA 4.S. Corner and R. Gobel, Presenting endomorphism algebras- a uniform treatment, Proc. London Math Soc..

[DMR] $\square$. van Douwen, J.D. Monk and M. Rabin, Some questions about Boolean algebras, Algebra Universatis 11 (1980), 220-243.

[GSR $\mathbb{R}$. Gobel and S. Shelah, Modules over arbitray domains, I, Math Zeitschift.

[GS2] _ Modules over arbitray domain,II, Fund. Math.

[M] D. Monk, A very rigid Boolean algebra, Israel J. Math no 35 (1980), 135-150.

[Sh1\$. Shelah, Clasification Theory and the number of non isomorphic models, (1978).

[Sh2] _ B Boolean algebras with few endomorphisms, Proc of A.M.S. no 14 (1979), $135-142$.

[Sh3]__ Existence of rigid like families of abelian p-groups, . Model Theory and Algebra (1975), 385-402.

[Sh4]__ A combinatorial principle and endomorphism rings of p-groups I Proc of the Jerusalem model theory year, Israel J. Math.

[Sh5]__ A combinatorial principle and endomorphism rings of abelian groups II, Proc of the Conference on Abelian groups (April 1984), Iudine,Italy.

[Sh6]_ Why there are many non-isomorphic models of unsuperstable theoreies,, Proc. of the International Congress of Math ,Vancouver (1974 pages 553-557).

[Sh7]__ Abstract of A.M.S. (1984). 